\title{
Constructing Lefschetz-type fibrations on four-manifolds
}

\author{
DAVID T GAY \\ ROBION KIRBY
}

\begin{abstract}
We show how to construct broken, achiral Lefschetz fibrations on arbitrary smooth, closed, oriented 4-manifolds. These are generalizations of Lefschetz fibrations over the 2-sphere, where we allow Lefschetz singularities with the non-standard orientation as well as circles of singularities corresponding to round 1-handles. We can also arrange that a given surface of square 0 is a fiber. The construction is easier and more explicit in the case of doubles of 4-manifolds without 3-and 4-handles, such as the homotopy 4-spheres arising from nontrivial balanced presentations of the trivial group.
\end{abstract}

57M50; 57R17

\section{Introduction}

Theorem 1.1 Let $X$ be an arbitrary closed 4-manifold and let $F$ be a closed surface in $X$ with $F \cdot F=0$. Then there exists a broken, achiral Lefschetz fibration (BALF) from $X$ to $S^{2}$ with $F$ as a fiber.

Recall that a (topological) Lefschetz fibration (LF) on a closed 4-manifold is a smooth map to a closed surface with all singularities locally modelled by the complex map $(w, z) \mapsto w^{2}+z^{2}$. (We call these "Lefschetz singularities".) An achiral LF (ALF) is one in which we also allow singularities modelled by $(w, z) \mapsto(\bar{w})^{2}+z^{2}$, the same model as above but with the opposite orientation on the domain. (We call these "anti-Lefschetz singularities".) All (anti-)Lefschetz singularities in this paper will be allowable, see Definition 2.3. A broken LF (BLF) is one in which we also allow singularities modelled by the map from $S^{1} \times \mathbb{R}^{3}$ to $S^{1} \times \mathbb{R}$ given by $(\theta, x, y, z) \mapsto\left(\theta,-x^{2}+y^{2}+z^{2}\right)$. (We call these "round 1-handle singularities".) Such a fibration was called a "singular LF" in Auroux-Donaldson-Katzarkov [4], and the singularities were called "indefinite quadratic singularities" there. Finally, a broken achiral LF (BALF) is one in which all three types of singularities are allowed.

This theorem can be compared to work of Auroux, Donaldson and Katzarkov [4] and of Etnyre and Fuller [12]. In the first it is shown that if $X^{4}$ has a near-symplectic form 
(which it does when $b_{2}^{+}>0$ ), then $X^{4}$ is a broken Lefschetz pencil (BLP). This is a generalization of Donaldson's earlier results on Lefschetz pencils and symplectic structures [9]. In particular, $X$ blown up some number of times is a Lefschetz fibration over each hemisphere of $S^{2}$ with different genus fibers, and then over the equator round 1-handles are added (independently) to the side with lower genus; also the Lefschetz singularities can all (topologically) be placed over the high genus hemisphere. In our paper, round 1-handles can also be added independently; see Addendum 1.3.

Etnyre and Fuller show that $X^{4}$ connected sum with a 2 -sphere bundle over $S^{2}$ is an achiral Lefschetz fibration (ALF); the connected sum occurs as the result of surgery on a carefully chosen circle in $X$. Baykur [6] has results relating this construction to folded symplectic structures.

Conjecture 1.2 Not all closed, smooth, oriented 4-manifolds are BLFs. For example, it is possible that $\mathbb{C} P^{2}$ is necessarily achiral as a fibration (even though it does have a Lefschetz pencil structure).

We also prove the following.

Addendum 1.3 (Addendum to Theorem 1.1) If we are given a collection of embedded 2-spheres $S_{1}, \ldots, S_{n}$, each intersecting $F$ in a single positive intersection, then we can construct the BALF so that each $S_{i}$ is a section. In particular, if the initial "fiber" $F$ has positive self-intersection, we can blow up its intersection points, make a BALF in which the exceptional divisors are sections, and then blow down these sections, to get a broken, achiral Lefschetz pencil (BALP) with $F$ as a fiber.

We can arrange that the round 1-handle singularities all project to the tropics of Cancer and Capricorn, with their high genus sides towards the equator and with all Lefschetz and anti-Lefschetz singularities over the equator.

A significant section of this paper is devoted to proving a result (Theorem 5.2 and Corollary 5.3) on the existence of "convex" BLFs on 4-manifolds built from 0-, 1and 2-handles, with prescribed boundary conditions. This is essential to the proof of Theorem 1.1, but is also of independent interest as a natural generalization of Loi and Piergallini's result [28] (see also Akbulut and Ozbagci [3]) on the existence of Lefschetz fibrations on Stein surfaces.

The virtues of Theorem 1.1 are as follows.

(1) It covers small 4-manifolds such as homology 4-spheres. In particular the Gluck construction on a knotted 2-sphere $K$ in $S^{4}$ is a possibly exotic homotopy 
4-sphere which is a BALF with $K$ as a fiber. Also, the homotopy $4-$ spheres arising from non-trivial presentations of the trivial group (see Kirby [25, Problem 5.1 and 5.2]) are seen by a simplified construction to be BALFs. $\mathbb{C} P^{2}$ with either orientation can be seen as a simple example of a BALF.

(2) The proof is fairly constructive, with the least constructive part coming from the use of Giroux's theorem that two open books on a 3-manifold are stably equivalent if their 2-plane fields are isotopic [17] and Eliashberg's theorem that homotopic overtwisted contact structures are isotopic [11].

(3) Conceivably these BALFs can be used as LFs are used in Donaldson-Smith theory [10] (and BLFs in Perutz's generalization $[30 ; 31 ; 32]$ ) to find multisections which are pseudoholomorphic curves, in the sense of Taubes' program [34;33] on pseudoholomorphic curves in near-symplectic 4-manifolds.

(4) In a philosophical sense, this paper complexifies Morse functions as much as possible, in the sense that it produces maps from arbitrary 4 -manifolds to $\mathbb{C} P^{1}$ which, locally, are as complex analytic as possible. This continues the long line of results (obtaining pencils) from Lefschetz ( $X$ algebraic) to Donaldson ( $X$ symplectic) to Auroux-Donaldson-Katzarkov ( $X$ near-symplectic).

This is an existence theorem, so of course there ought to be a uniqueness theorem, which we hope will be the subject of a following paper.

Remark 1.4 After we posted our initial version of this paper, Perutz pointed out that a simple argument in [4, Section 8.2, Example 3] can, with a reversal of orientations, be used to turn an anti-Lefschetz singularity into a round 1-handle singularity at the cost of blowing up the 4-manifold. Thus a corollary of Theorem 1.1 is that, after some number of blowups, any closed 4-manifold has a BLF over $S^{2}$. We will present our version of this construction in Remark 6.2 at the end of the paper. Note here that the exceptional divisors are not sections of the BLF, and that the round 1-handle singularities do not all project to parallel copies of the equator, so this does not quite recover the main result of [4]. However, this construction may be sufficient as input into Perutz's program to construct smooth invariants from BLFs.

We would especially like to thank the African Institute of Mathematical Sciences in Cape Town for their hospitality during the final writing of this paper. We are also grateful to the referee for catching some important and subtle errors.

David Gay was supported in part by NSF/DMS-0244558 and fellowships from CRM/ ISM and CIRGET. Robion Kirby was supported in part by NSF/DMS-0244558. 


\subsection{Outline}

We begin in Section 2 by giving precise definitions of the types of fibrations considered, including control on behavior near singularities and along boundaries. While doing this, we also show how to achieve the singularities and boundary behavior in terms of handle additions, and we show how such handle additions affect the monodromies of fibrations and open book decompositions (OBDs) on the boundaries. The two important types of boundary behavior we define are "convexity" and "concavity" along boundaries, conditions which mean that the fibrations restrict to OBDs on the boundary and that concave boundaries can be glued to convex boundaries as long as the OBDs match. The proof of Theorem 1.1 then boils down to constructing a concave piece and a convex piece and arranging that the open books match.

In Section 3 we look in detail at an example from [4] of a BLF on $S^{4}$, breaking it down into handles as in Section 2. The goal is to get the reader accustomed to the tools and language we use in the rest of the paper, and to see various ways to split the BLF into convex and concave pieces. In particular we show (Lemma 3.1) how to construct a concave BLF on $F \times B^{2}$ for any closed surface $F$.

In Section 4 we show how to construct a BALF on the double of any 4-dimensional 2-handlebody. This construction is more explicit than the general case because it does not depend on Giroux's work on open books or Eliashberg's classification of overtwisted contact structures. This section also includes a method (Lemma 4.5) for adding 1handles to a concave (BA)LF. At the end of the section we discuss the relationship between doubles and the Andrews-Curtis conjecture about balanced presentations of the trivial group.

Then in Section 5 we show that a 4-manifold $X$ built from just $0-, 1-$ and 2-handles is a convex BLF. Furthermore, if we are given a homotopy class of plane fields on $\partial X$, we can arrange that the induced OBD on $\partial X$ supports an overtwisted contact structure in this homotopy class. (This is not true for ALFs.) In order to achieve this, we need to be able to positively and negatively stabilize the OBD on $\partial X$. (Stabilization means plumbing on Hopf bands, positive being left-handed bands and negative being right-handed bands.) Positive stabilization is easy to achieve; negative stabilization is easy if we allow achirality, but to avoid achirality as much as possible we show in Lemma 5.4 that we can negatively stabilize with round 1-handles instead of achiral vanishing cycles. This section also includes a detailed analysis of almost complex structures carried by BLFs.

Section 6 finishes off the proof of Theorem 1.1 and the addendum. We take the concave BLF on $F \times B^{2}$ from Section 3 and add enough 1-handles (as in Section 4) so that the 
complement is built with just $0-, 1-$ and 2 -handles. This induces a particular OBD on the boundary of this concave piece. We then construct a convex BLF on the complement as in Section 5, inducing an OBD on its boundary which supports a contact structure homotopic to the contact structure supported by the OBD coming from the concave piece. We arrange that both contact structures are overtwisted, so by Eliashberg's classification of overtwisted contact structures [11] they are isotopic. By Giroux's work on open books [17] the two OBDs have a common positive stabilization, which we already know we can achieve on the convex piece without introducing achirality. (Note that at this point the two pieces are BLFs, not BALFs.) The only new tool developed in this section is a trick for stabilizing OBDs on concave boundaries of (BA)LFs; unfortunately, to achieve the positive stabilizations we are forced to introduce anti-Lefschetz singularities (achirality).

Section 7 gives a list of questions.

\subsection{Notation and conventions}

Unless otherwise stated, all manifolds are smooth, compact, connected and oriented (possibly with boundary), and all maps between manifolds are smooth. Whenever we specify a local model for the behavior of a map, we imply that the local models respect all orientations involved. All almost complex structures respect orientations and all contact structures are positive and co-oriented.

For our purposes, an open book decomposition (OBD) on a closed 3-manifold $M$ is a smooth map $f: M \rightarrow B^{2}$ such that $f^{-1}\left(\partial B^{2}\right)$ is a compact 3-dimensional submanifold on which $f$ is a surface bundle over $S^{1}=\partial B^{2}$ and such that the closure of $f^{-1}\left(B^{2} \backslash \partial B^{2}\right)$ is a disjoint union of solid tori on each of which $f$ is the projection $S^{1} \times B^{2} \rightarrow B^{2}$. The binding is $B=f^{-1}(0)$, and the page over $z \in S^{1}$ is $\Sigma_{z}=$ $f^{-1}\{\lambda z \mid 0 \leq \lambda \leq 1\}$, with $B=\partial \Sigma_{z}$. The monodromy is the isotopy class (rel. boundary) of the return map $h: \Sigma_{1} \rightarrow \Sigma_{1}$ for any vector field transverse to the interiors of all the pages and meridinal near the binding. We will usually blur the distinction between the isotopy class and its representatives. Positively (resp. negatively) stabilizing an OBD $f: M \rightarrow B^{2}$ means plumbing on a left-handed (resp. right-handed) Hopf band. Thus if $f^{\prime}: M \rightarrow B^{2}$ is the result of positively (resp. negatively) stabilizing $f: M \rightarrow B^{2}$, then $f^{\prime}:-M \rightarrow B^{2}$ is the result of negatively (resp. positively) stabilizing $f:-M \rightarrow B^{2}$.

When a knot $K$ lies in a page of an open book decomposition or a fiber of a fibration over $S^{1}$, we call the framing induced by the page the "page framing", and abbreviate it $\operatorname{pf}(K)$. 


\section{Broken, achiral Lefschetz fibrations and pencils}

We will be constructing and working with smooth surjective maps from compact 4-manifolds to compact surfaces with controlled behavior at singularities and along boundaries, this control to be discussed below. When such a map $f: X^{4} \rightarrow \Sigma^{2}$ is defined on all of $X$ we will call $f$ a "fibration", decorated with various adjectives which characterize the allowed singularities and boundary behavior. When $f$ is defined only on the complement of a discrete set $B \subset X$, near each point of which $f$ is locally modelled by the canonical map $\mathbb{C}^{2} \backslash 0 \rightarrow \mathbb{C} P^{1}$, we will call $f$ a "pencil", decorated with the same adjectives; the points of $B$ are called "base points". Note that for a pencil the target surface $\Sigma$ is necessarily $S^{2}$. Also note that blowing up each base point turns a pencil into a fibration, with the exceptional divisors becoming sections. Similarly, blowing down square -1 sections of a fibration over $S^{2}$ yields a pencil. If $f: X \backslash B \rightarrow \Sigma^{2}$ is a pencil and $p \in S^{2}$, we abuse terminology slightly to say that the "fiber" over $p$ is $f^{-1}(p) \cup B$, a compact surface, so that any two fibers intersect transversely and positively at each base point.

Now we describe the adjectives which characterize the singularities, as well as interpretations of the singularities in terms of handlebody decompositions and the effects of the various singularities on monodromies of fibrations on boundaries. The relationships between singularities, handles and monodromies are critical for all the constructions in this paper.

Consider a general smooth map $f$ from a 4 -manifold $X$ to a surface $\Sigma$.

Definition 2.1 A critical point $p \in X$ of $f$ is a Lefschetz singularity if $f$ is locally modelled near $p$ by the map $g:(w, z) \mapsto w^{2}+z^{2}$ from $\mathbb{C}^{2}$ to $\mathbb{C}$. If instead $f$ is locally modelled near $p$ by $g \circ \tau$, where $\tau(w, z)=(\bar{w}, z)$ reverses orientation, then $p$ is an anti-Lefschetz singularity.

A Lefschetz singularity is the standard singularity in a Lefschetz fibration, corresponding to the critical point of a vanishing cycle. The following remark is a standard result and, if the reader finds it confusing, a more detailed exposition can be found in Gompf and Stipsicz [20].

Remark 2.2 (Vanishing cycles as 2 -handles) If $[0,1] \times S^{1}$ is an annulus in $\Sigma$ with a single Lefschetz singularity in $f^{-1}\left([0,1] \times S^{1}\right)$, then $f^{-1}\left([0,1] \times S^{1}\right)$ is a cobordism from $M_{0}=f^{-1}\left(0 \times S^{1}\right)$ to $M_{1}=f^{-1}\left(1 \times S^{1}\right)$ on which the projection to [0,1] is a Morse function with a single Morse critical point of index 2 (at the Lefschetz singularity). The corresponding 2-handle is attached along a knot $K$ in $M_{0}$ which 
in fact lies in a fiber of the fibration of $M_{0}$ over $S^{1}$, and the framing is one less than the framing induced by the fiber, ie $\operatorname{pf}(K)-1$. Conversely, suppose we start with a fibration $f: X^{4} \rightarrow \Sigma^{2}$, where $\Sigma$ has nonempty boundary and $f$ has no singularities over $\partial \Sigma$. Now attach a 2 -handle to $X$ along a knot $K$ in a fiber of the fibration $f^{-1}(\partial \Sigma) \rightarrow \partial \Sigma$, with framing $\operatorname{pf}(K)-1$, to make a new 4-manifold $X^{\prime} \supset X$. Then $f$ extends to a fibration of $X^{\prime}$ over $\Sigma$ with exactly one new singularity, a Lefschetz singularity, at the core of the 2-handle. Lastly, if the monodromy of the fibration on $\partial X$ is $h$ and the monodromy of the fibration on $\partial X^{\prime}$ is $h^{\prime}$, the relation is that $h^{\prime}=\tau_{K} \circ h$, where $\tau_{K}$ is a right-handed Dehn twist along $K$.

If instead we started with an anti-Lefschetz singularity, the 2-handle would be attached with framing $\operatorname{pf}(K)+1$ and, conversely, if we attach a 2 -handle as above but with framing $\operatorname{pf}(K)+1$ rather than $\operatorname{pf}(K)-1$, we can extend the fibration creating a single new anti-Lefschetz singularity, and the monodromy changes by a left-handed Dehn twist (ie $h^{\prime}=\tau_{K}^{-1} \circ h$ ).

Definition 2.3 An (anti-)Lefschetz singularity is allowable if the attaching circle of its vanishing cycle is homologically nontrivial in the fiber.

As preamble to the next definition, recall that a "round $k$-handle" is $S^{1}$ times a $k$-handle. Thus a 4-dimensional round 1-handle is $S^{1} \times B^{1} \times B^{2}$ attached along $S^{1} \times S^{0} \times B^{2}$, ie attached along a pair of oriented framed knots. It is not hard to see that the only important data is the relative orientation of the pair (if we reverse one knot we should reverse the other) and the relative framing (if we increase one framing by $k$ we should decrease the other by $k$ ). A round 1-handle can also be thought of as a 1-handle and a 2-handle, with the attaching circle for the 2-handle running geometrically twice and algebraically zero times over the 1 -handle. We will either draw round 1-handles this way, or shrink the balls of the 1-handles down to small solid black disks, so that we see two framed knots each decorated with a big black dot, and a dashed line connecting the two dots. Drawn this latter way, it is important to indicate the orientations with arrows. Since only the relative framing matters, we will only label one of the two knots with a framing, implying that the other is 0 -framed. If a 2-handle runs over a round 1-handle, we see its attaching circle as an arc or sequence of arcs starting and ending on the attaching circles for the round 1-handle. Figure 1 gives two drawings of a handlebody decomposition of $B^{4}$ involving a 1-handle, a round 1 -handle and a 2 -handle.

Definition 2.4 An embedded circle $S \subset X$ of critical points of $f$ is a round 1handle singularity if $f$ is locally modelled near $S$ by the map $h:(\theta, x, y, z) \mapsto$ $\left(\theta,-x^{2}+y^{2}+z^{2}\right)$ from $S^{1} \times \mathbb{R}^{3}$ to $S^{1} \times \mathbb{R}$. Note that the genus of a fiber on one 

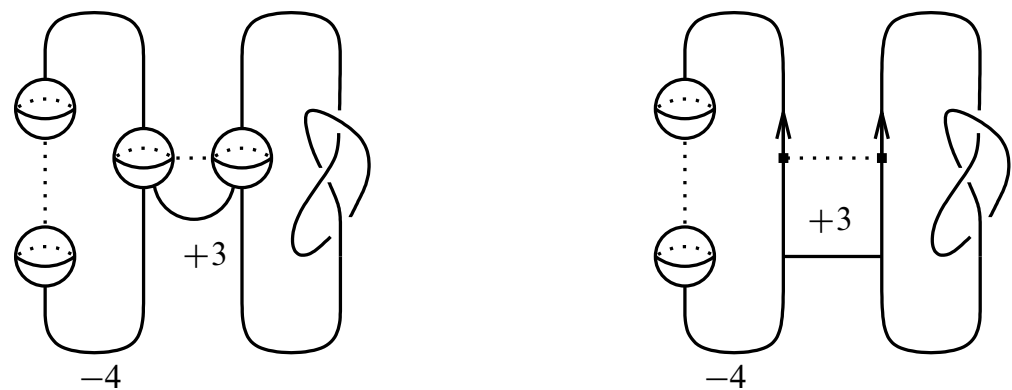

Figure 1: Two drawings of a handlebody decomposition of $B^{4}$ involving a 1 -handle, a round 1-handle and a 2-handle; on the left the round 1-handle is drawn as a 1 -handle and a 2 -handle.

side of $f(S)$ is one higher than the genus on the other side; we will refer to these as the high-genus side and the low-genus side.

This type of singularity is called an "indefinite quadratic singularity" in [4], which in principle also allows for a local model which is a quotient of the above model by a $\mathbb{Z} / 2$ action so that the annulus $\{y=z=0\}$ becomes a Möbius band. In this paper we do not need this nonorientable model.

Remark 2.5 (Attaching round 1-handles) Let $[0,1] \times S^{1}$ be an annulus in $\Sigma$ with a single round 1-handle singularity $S$, and no other singularities, in $f^{-1}\left([0,1] \times S^{1}\right)$, with $f(S)=1 / 2 \times S^{1}$, and with the low genus side over $0 \times S^{1}$ and the high genus side over $1 \times S^{1}$. Then $f^{-1}\left([0,1] \times S^{1}\right)$ is a cobordism from $M_{0}=f^{-1}\left(0 \times S^{1}\right)$ to $M_{1}=f^{-1}\left(1 \times S^{1}\right)$ which is the result of attaching a round 1 -handle to $M_{0}$ along a framed, oriented pair of knots $\left(K_{1}, K_{2}\right)$ each of which is a section of the fibration over $S^{1}$, ie each one is transverse to all the fibers and wraps once around the fibration in the positive direction. Conversely, if we start with a fibration $f: X \rightarrow \Sigma$ with no singularities in $f^{-1}(\partial \Sigma)$, if we choose any such pair $\left(K_{1}, K_{2}\right)$ in $f^{-1}(\partial \Sigma)$, and if we attach a round 1 -handle along $\left(K_{1}, K_{2}\right)$ to produce a new 4-manifold $X^{\prime} \supset X$, then $f$ extends to $f^{\prime}: X^{\prime} \rightarrow \Sigma$ with one new round 1-handle singularity the image of which is parallel to $\partial \Sigma$, and no other new singularities. The fibers in $\partial X^{\prime}$ are the result of 0 -surgery on the fibers in $\partial X$ at the two points where $K_{1}$ and $K_{2}$ intersect the fibers. To see how the monodromy changes, consider a vector field transverse to the fibers in $\partial X$ with $K_{1}$ and $K_{2}$ as closed orbits such that the return map $h$ on a fiber $F$ fixes a disk neighborhood $D_{i}$ of each $F \cap K_{i}$ and such that closed orbits close to $K_{1}$ and $K_{2}$ represent the framings with which we are to attach the round 1-handle. 
Let $F^{\prime}$ be the new fiber obtained by replacing $D_{1} \cup D_{2}$ by $[0,1] \times S^{1}$. Then the new monodromy is equal to $h$ on $F \backslash\left(D_{1} \cup D_{2}\right)$ and the identity on $[0,1] \times S^{1}$.

Since a round 1-handle turned upside down is a round 2-handle, we could also understand constructions with round 1-handle singularities in terms of round 2-handles. However, in our proofs we do not seem to need this perspective.

Definition 2.6 The adjective "Lefschetz" is used to mean that a given map (fibration or pencil) is allowed to have Lefschetz singularities. We add the adjective "achiral" to "Lefschetz" to indicate that we allow both Lefschetz and anti-Lefschetz singularities (recall that these are always allowable, as in Definition 2.3). The adjective "broken" means that round 1-handle singularities are allowed. (This term is due to Perutz [30] and Smith and has been chosen to indicate that the non-singular fibers change genus when moving across the image in the base of a round 1-handle singularity; since the singular circles disconnect the base, these singularities "break" the fibration in a certain sense.) If a type of singularity is not explicitly allowed then it is forbidden.

To summarize and abbreviate, we have four kinds of "fibrations": Lefschetz fibrations (LFs), achiral Lefschetz fibrations (ALFs), broken Lefschetz fibrations (BLFs) and broken achiral Lefschetz fibrations (BALFs), with containment as follows: $L F \subset A L F$, $L F \subset B L F, A L F \subset B A L F$ and $B L F \subset B A L F$. Replacing "fibration" with "pencil" and "F" with "P" in the preceding sentence also works.

Now we describe the kind of boundary behavior we will allow for fibrations and pencils on 4-manifolds with nonempty boundary. Again consider a general smooth map $f: X^{4} \rightarrow \Sigma^{2}$, and now let $M^{3}$ be a component of $\partial X$.

Definition 2.7 We say that $f$ is "flat" along $M$ if $f(M)$ is a component of $\partial \Sigma$ and if $\left.f\right|_{M}$ is an honest fibration over this component. We say that $f$ is "convex" along $M$ if $f(M)=\Sigma=B^{2}$ and if $\left.f\right|_{M}: M \rightarrow B^{2}$ is an open book decomposition of $M$. We say that $f$ is "concave" along $M$ if $f(M)$ is a disk $B^{2}$ in the interior of $\Sigma$ and if $\left.f\right|_{M}$ is an open book decomposition of $M$. If $f$ is flat (resp. convex or concave) along each component of $\partial X$, we simply say that $f$ is flat (resp. convex or concave).

Note that, for a convex fibration, the fibers are surfaces with boundary. We use the term "convex" because a convex Lefschetz fibration with "allowable" vanishing cycles (homologically nontrivial in the fiber) naturally carries a symplectic structure (in fact, a Stein structure) which has convex boundary. Likewise, a concave Lefschetz pencil carries a symplectic structure with concave boundary; in this case some fibers are closed and some are compact with boundary. The term "flat" is similarly motivated; here the fibers are all closed. 
The typical example of a convex (BA)LF is $F \times B^{2}$ where $F$ is a surface with nonempty boundary, together with vanishing cycles (maybe of both kinds) and round 1-handles.

Remark 2.8 (Convex 1-handles and concave 3-handles) Suppose that $f: X \rightarrow B^{2}$ is a convex fibration and that $X^{\prime}$ is the result of attaching a 1 -handle to $X$ at two balls $B_{0}, B_{1}$ which are "strung on the binding" of the induced OBD on $\partial X$ in the sense that $\left.f\right|_{B_{i}}$ is the standard projection $B^{3} \rightarrow B^{2}$. Then $f$ extends to a convex fibration $f^{\prime}: X^{\prime} \rightarrow B^{2}$ with no new singularities. Each fiber $F^{\prime}$ of $f^{\prime}$ is diffeomorphic to a fiber $F$ of $f$ with a 2-dimensional 1 -handle attached along the two intervals $\partial F \cap B_{0}$ and $\partial F \cap B_{1}$, and the same relation holds between the pages of the new OBD on $\partial X^{\prime}$ and the pages of the old OBD on $\partial X$. The new monodromy is the old monodromy extended by the identity across the 1 -handle.

Dually, if $f: X \rightarrow \Sigma^{2}$ is a concave fibration and $X^{\prime}$ is the result of attaching a 3handle to $X$ along a 2-sphere $S$ such that $\left.f\right|_{S}$ is the standard projection $S^{2} \rightarrow B^{2}$, then $f$ extends to a concave fibration $f^{\prime}: X^{\prime} \rightarrow \Sigma$ with no new singularities. Each page $F^{\prime}$ of the new OBD on $\partial X^{\prime}$ is diffeomorphic to a page $F$ of $\partial X$ cut open along the arc $S \cap F$. Implicit here is that the old monodromy was trivial in a neighborhood of this arc, and so the new monodromy is just the old monodromy restricted to $F^{\prime}$. The fibers of $f^{\prime}$ are related to the fibers of $f$ as follows: If $f(\partial X)=B^{2} \subset \Sigma$, then the fibers over $\Sigma \backslash B^{2}$ do not change, while the fibers of $f^{\prime}$ over points in $B^{2}$ are obtained from the fibers of $f$ over the same points by attaching 2-dimensional 1-handles. The subtle point here is that each fiber of the fibration inside the 4-manifold gains a 1-handle while each fiber of the OBD on the boundary loses a 1-handle.

Remark 2.9 Some other handle attachments that are not used in this paper but that can help develop the reader's intuition are as follows: If one attaches 2-handles to a convex (BA)LF, with one 2-handle attached along each component of the binding of the induced open book, with framings 0 relative to the pages, one produces a flat (BA)LF. Using +1 framings instead produces a concave (BA)LP (Gay [15]).

Remark 2.10 (From flat to concave) One way to construct a concave (BA)LF is to start with a flat (BA)LF and attach one or more 2-handles along sections of the surface bundle induced on the boundary. More concretely, suppose that $f: X \rightarrow \Sigma$ is flat along a boundary component $M \subset \partial X$ and that $K_{1}, \ldots, K_{n}$ are framed knots in $M$ which are sections of the induced fibration $f: M \rightarrow S^{1} \subset \partial \Sigma$. Let $X^{\prime} \supset X$ be the result of attaching 2-handles along $K_{1}, \ldots, K_{n}$ to $X$, and let $M^{\prime}$ be the new boundary component coming from surgery on $M$. Then $f$ extends to $f^{\prime}: X^{\prime} \rightarrow \Sigma^{\prime}$, where $\Sigma^{\prime}$ is the result of attaching a disk $D$ to the relevant component of $\partial \Sigma$, so that $f^{\prime}$ is concave along $M^{\prime}$. The cores of the 2-handles become sections of $f^{\prime}$ over $D$, 
which extend as sections over all of $X^{\prime}$ as long as the knots $K_{i}$ extend as sections of $f$ over all of $X$. A concave (BA)LF which is used later in this paper is obtained simply from $F \times B^{2}, F$ a closed surface, together with a 2-handle added to point $\times S^{1}$ with framing 0 .

In this process we transform a surface bundle over $S^{1}$ on $\partial X$ into an OBD on $\partial X^{\prime}$. Each page of the new OBD is diffeomorphic to a fiber of the fibration on $\partial X$ with a disk removed at each point of intersection with the sections $K_{1}, \ldots, K_{n}$. If we choose a vector field $V$ transverse to the fibers in $\partial X$ such that each $K_{i}$ is a closed orbit with a neighborhood $v_{i}$ of closed orbits realizing the given framing of $K_{i}$, and if $h$ is the return map on a fiber $F$ for flow along $V$, then the monodromy of the new OBD on $\partial X^{\prime}$ is precisely $h$ restricted to the new page $F \backslash\left(D_{1} \cup \cdots \cup D_{n}\right)$, where $D_{i}=v_{i} \cap F$.

Remark 2.11 (Glueing fibrations and pencils along boundaries) The point of spelling out the above boundary conditions is that it should now be clear that fibrations and pencils can be glued along common boundaries as long as we either

(1) glue flat boundaries to flat boundaries via orientation reversing diffeomorphisms respecting the induced fibrations over $S^{1}$ or

(2) glue convex boundaries to concave boundaries via orientation-reversing diffeomorphisms respecting the induced open book decompositions.

\section{The Auroux-Donaldson-Katzarkov 4-sphere}

In [4, Section 8] on singular (or broken) Lefschetz fibrations, Auroux, Donaldson and Katzarkov construct a BLF $f: S^{4} \rightarrow S^{2}$. The fiber over the north pole is $S^{2}$, and over the south pole is $T^{2}$. Over the polar caps are $S^{2} \times B^{2}$ and $T^{2} \times B^{2}$. A round 1 -handle is attached to $S^{2} \times B^{2}$, giving a new boundary equal to $T^{2} \times S^{1} \rightarrow S^{1}$. Now this is glued to $T^{2} \times B^{2} \rightarrow B^{2}$ by a diffeomorphism of $T^{2} \times S^{1}$ which rotates $T^{2}$ along a meridian as $S^{1}$ is traversed, ie by a matrix of the form

$$
\left(\begin{array}{lll}
1 & 0 & 1 \\
0 & 1 & 0 \\
0 & 0 & 1
\end{array}\right)\left(\begin{array}{l}
s \\
t \\
\theta
\end{array}\right)=\left(\begin{array}{c}
s+\theta \\
t \\
\theta
\end{array}\right), \quad \theta \in S^{1} .
$$

The complement of the preimage $S^{2} \times B^{2}$ of the arctic cap is an interesting BLF for $B^{3} \times S^{1} \rightarrow B^{2}$ restricting to $S^{2} \times S^{1} \rightarrow S^{1}$ on the boundary; it is made from $T^{2} \times B^{2}$ by adding a round 2 -handle in the right way.

However, it is more useful to describe the BLF in a somewhat different way. If we pick the 0 -handle and one of the 1 -handles in $T^{2}$, then its thickening gives 
$[0,1] \times S^{1} \times B^{2} \rightarrow B^{2}$, a convex fibration with fiber an annulus. The base $B^{2}$ will become the southern hemisphere $D_{S}$ of $S^{2}$. The complement in $S^{4}$ must be $S^{2} \times B^{2}$, with a smaller $S^{2} \times B^{2}$ in its interior mapped by projection $S^{2} \times B^{2} \rightarrow B^{2}$ into the northern hemisphere $D_{N}$. The fibration on this smaller $S^{2} \times B^{2}$ is then flat along its boundary, inducing the fibration $S^{2} \times S^{1} \rightarrow S^{1}$. The cobordism in between, $S^{2} \times S^{1} \times I$, will be mapped into $S^{2}$ in a way described below, with one concave boundary component and one flat boundary component which match the convex and flat boundaries of the two pieces constructed above.

The cobordism $S^{2} \times S^{1} \times I$ can be written as a cancelling 1-2-handle pair and a cancelling 2-3-handle pair, attached to $S^{2} \times B^{2}$ and not changing its diffeomorphism type. The 1-handle from the first pair and the 2-handle from the second pair will form a round 1-handle, attached trivially along a pair of circles $\left\{p_{1}, p_{2}\right\} \times \partial B^{2} \subset \partial\left(S^{2} \times B^{2}\right)$, and mapping down to $D_{N}$. (The fibration extends over this 1 -handle as in Remark 2.5).

The remaining 2-handle and 3-handle in fact make up a round 2-handle, or dually a round 1 -handle attached to the thickened annulus $[0,1] \times S^{1} \times B^{2}$, since the complement of an annulus in $T^{2}$ is an annulus, and adding an annulus is the same as adding a round 1-handle. However, we do not use it as a round 2-handle here, but rather we map the 2-handle and 3-handle down to $D_{S}$ as follows.

A handlebody picture of the process is given in Figure 2. The 2-handle labelled $H$ is the 2-handle of the round 2-handle in the preceding paragraph, and in the figure we see that its attaching map is a section of the fibration over $S^{1}$, so that fibration will extend over $H$ exactly as in Remark 2.10. Here, the framings of the 2-handles are chosen so that when the 2 -handle in the round 1 -handle is slid twice over $H$ (see Figure 3), then it becomes an unknot, separated from the other components, with framing 0 , so that it defines a 2 -sphere to which the 3 -handle (in the round 2-handle) is attached. $H$ then cancels the remaining 1 -handle.

There are several features about this construction that should be noted. First, the concave piece has been constructed by adding the 2-handle $H=B^{2} \times B^{2}$ along a section of $T^{2} \times S^{1} \rightarrow S^{1}=\partial D_{N}$ which does not (in this case) extend over $D_{N}$, and which maps to $D_{S}$ by projection on the first factor. The fact that the section does not extend to $D_{N}$ is necessary for otherwise $S^{4}$ would contain a hyperbolic pair, the fiber and the global section. This is a key to finding BALFs for all homology 4-spheres. In particular, Theorem 1.1 shows that any knotted 2-sphere $K$ in $S^{4}$ can be made the fiber of a BALF on $S^{4}$. Then, after performing the Gluck construction on $K$, the resulting homotopy $4-$ sphere is seen to be a BALF with fiber still equal to $K$. 


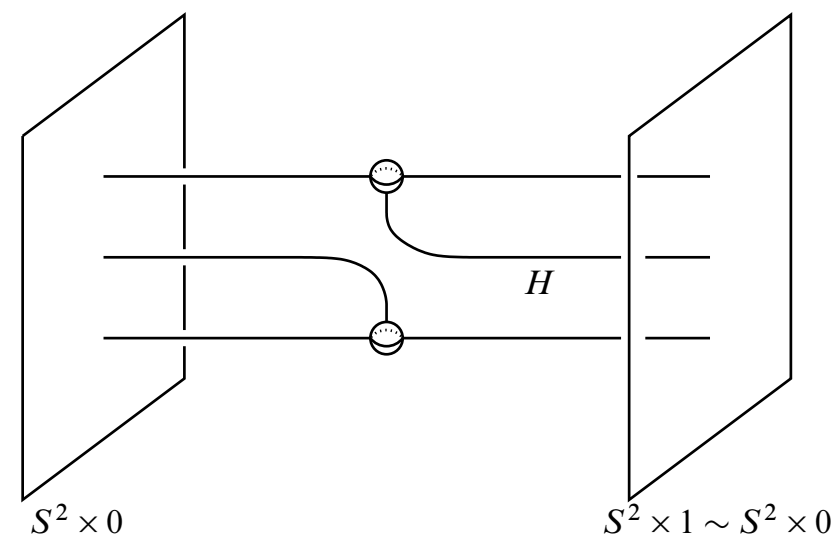

Figure 2: Finding an $H$.

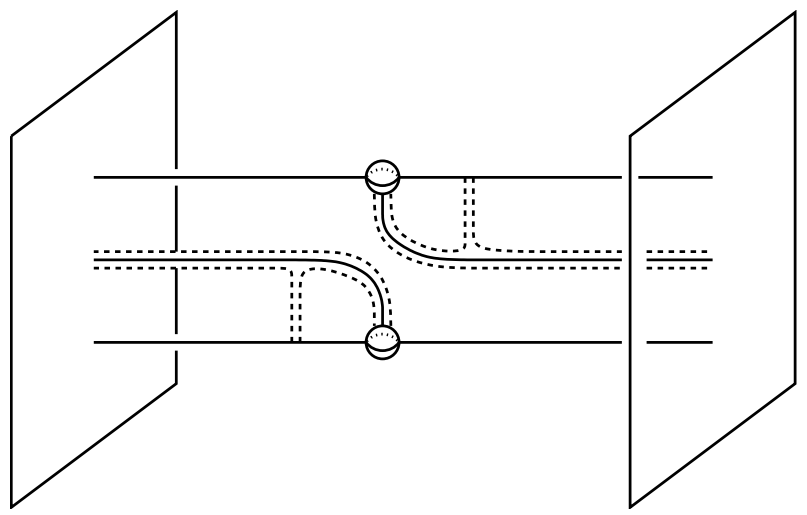

Figure 3: Sliding twice over $H$.

Second, the 3-handle of the round 2-handle (the 2-handle being $H$ ) is in a sense attached upside down to the concave side, as in Remark 2.8; the attaching 2-sphere consists of a pair of disks parallel to $H$ and a cylinder $S^{1} \times I$ which is attached to a circle family of arcs in the fibers of $T^{2} \times S^{1} \rightarrow \partial D_{N}$.

Third, it is not necessary to begin building the concave piece with a $2-$ sphere fiber. Instead begin with $F^{2} \times B^{2} \rightarrow B^{2}=D_{N}$, where $F$ is a closed surface of genus $g$. Pick a pair of points $p_{1}, p_{2} \in F$ and attach a round 1-handle along the sections $\left\{p_{1}, p_{2}\right\} \times \partial B^{2}$ over $\partial D_{N}$. Now add $H$ and the 3 -handle as before, and all the handles cancel topologically. (Figure 2 and Figure 3 are the same except that the squares at either end represent disks in $F$.) We have thus proved the following lemma. 
Lemma 3.1 Given any closed surface $F$ there exists a concave $B L F f: F \times B^{2} \rightarrow S^{2}$.

Note, however, that this statement of the result is deliberately vague about the resulting OBD on $F \times S^{1}=\partial\left(F \times B^{2}\right)$; this is because we will not need to know anything about the OBD when we use it later. However, in this $S^{4}$ example, it is important to see the OBD, and it is instructive to think about what happens with higher genus fibers.

Before adding the 3-handle, the boundary is an open book with a once-punctured fiber of genus $g+1$, called $F_{0}$. This open book does not have trivial monodromy when $g+1 \geq 2$, a fact that needs explaining. It is easiest to understand the monodromy after attaching $H$ if $H$ is added to a circle which corresponds to a fixed point of the monodromy before attaching $H$; see Remark 2.10. In this case, the initial monodromy is trivial, but $H$ is added to a curve representing the sum of the class of $\{p\} \times S^{1}$ in $\left(F \sharp\left(S^{1} \times S^{1}\right)\right) \times S^{1}$ and the class of a curve running over the first factor in $S^{1} \times S^{1}$, which we call $\alpha$. To adjust for this fact, monodromy is introduced along two curves $\alpha_{L}$ and $\alpha_{R}$ parallel to $\alpha$ which have the point $p$ between them, with a left twist $\tau_{\alpha_{L}}^{-1}$ on one and a right twist $\tau_{\alpha_{R}}$ on the other. Then the open book can be represented, as in Figure 4, by a fixed surface $F_{0}$ (obtained by removing a disk neighborhood of $p$ from $\left.F \sharp\left(S^{1} \times S^{1}\right)\right)$ with twists along the curves $\alpha_{L}$ and $\alpha_{R}$ drawn. When $g=0$ as in the case of $S^{4}$ above, then $\alpha_{L}$ and $\alpha_{R}$ are isotopic in $F_{0}$ so that the two twists cancel and the monodromy is still trivial after attaching $H$. But when $g>0, \alpha_{L}$ and $\alpha_{R}$ are not isotopic in $F_{0}$, so this construction gives a concave BLF whose boundary is an open book with non-trivial monodromy. The 3 -handle is then attached along the 2-sphere which intersects each page in the arc $\gamma$, so that $\alpha_{L}$ and $\alpha_{R}$ become boundary parallel Dehn twists. It follows that the convex piece, in order to fit with the concave piece, cannot be just a $(g+1)$-genus surface minus an annulus, crossed with $B^{2}$, for that has trivial monodromy on its boundary. However, if two vanishing cycles were added to the convex side along $\alpha_{L}$ and $\alpha_{R}$ (one framed $\mathrm{pf}+1$ and one framed $\mathrm{pf}-1$ ), this would produce a convex piece which would "dock" into the concave piece.

Having given the construction for $S^{4}$, it is now easy to describe a BALF on $\mathbb{C} P^{2}$. Simply take the above BLF for $S^{4}$ and add a +1 -framed 2-handle to the $T^{2} \times B^{2}$ along a nontrivial circle in the fiber on the boundary. This produces a single antiLefschetz singularity. The same construction with -1 gives us a BLF on $\overline{\mathbb{C} P^{2}}$. This is interesting because $\mathbb{C} P^{2}$ is symplectic (and is therefore a Lefschetz pencil) but seems to require achirality when described as a fibration, while $\overline{\mathbb{C} P^{2}}$ is far from symplectic but can be described as a fibration without using anti-Lefschetz singularities. 


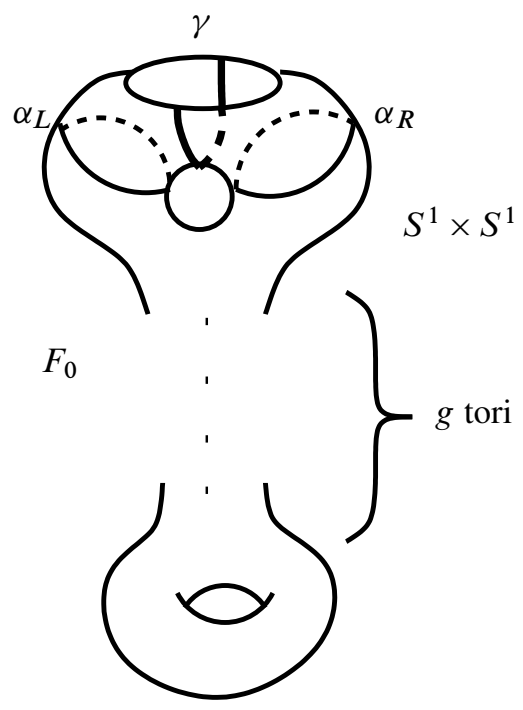

Figure 4: Monodromy after attaching $H$.

\section{Doubled 4-manifolds as BALFs}

In this section we will prove a simpler version of Theorem 1.1, namely that the double $D X$ of any 4-dimensional 2-handlebody $X$ is a BALF over $S^{2}$. Along the way we prove some important lemmas needed for the full proof of Theorem 1.1, but this simpler result has the nice feature of being more explicit than the full result in the sense that it does not rely on Giroux's work on open books or Eliashberg's classification of overtwisted contact structures.

The first tool we need is standard (see [3], for example).

Lemma 4.1 Suppose that $f: X \rightarrow B^{2}$ is a convex fibration and that $A$ is a properly embedded arc in a page of the induced $O B D$ on $\partial X$. First attach a 1-handle to $X$ at the two endpoints of $A$ and extend $f$ across the 1-handle as in Remark 2.8. Let $K$ be the knot lying in a page obtained by connecting the endpoints of $A$ by going over the 1handle, and now attach a 2-handle along $K$ with framing $\operatorname{pf}(K)-1(\operatorname{resp} \cdot \operatorname{pf}(K)+1)$ and extend $f$ across the 2-handle as in Remark 2.2. Since the 2-handle cancels the 1-handle we get a new BALF on $X$ with one more Lefschetz (resp. anti-Lefschetz) singularity (and different fibers). Then the new $O B D$ on $\partial X$ is the original $O B D$ with a left-handed (resp. right-handed) Hopf band plumbed on along $A$.

For clarification, recall that a Lefschetz singularity corresponds to a right-handed Dehn twist, which in the lemma above corresponds to a left-handed Hopf band (positive 
stabilization). Similarly, an anti-Lefschetz singularity corresponds to a left-handed Dehn twist, which in the lemma above corresponds to a right-handed Hopf band (negative stabilization).

Definition 4.2 Given a handlebody decomposition of a manifold $X$, let $X_{(k)}$ denote the union of handles of index less than or equal to $k$. We call $X$ a $k$-handlebody if $X=X_{(k)}$.

We will make essential use of the following proposition.

Proposition 4.3 (Harer [21], Akbulut-Ozbagci [3]) Given a 4-dimensional 2handlebody $X$, let $L$ be the attaching link for the 2-handles in $\partial X_{(1)}$. Then there exists a convex $L F f: X_{(1)} \rightarrow B^{2}$ such that $L$ lies in the interior of a single page $F$ of the induced open book decomposition of $\partial X_{(1)}$. Furthermore, it can be arranged that each component $K$ of $L$ can be connected to $\partial F$ by an arc $A \subset F$ avoiding $L$ (ie the interior of $A$ is disjoint from $L$ ).

Proof We do not need the full strength of the result in [3], so here we provide a streamlined proof of the result as we need it. The key fact we need is that if the page of an OBD of $S^{3}$ is obtained by plumbing left-handed Hopf bands onto a disk [22], then this OBD is induced by a Lefschetz fibration on $B^{4}$. (Start with the fibration $B^{4}=B^{2} \times B^{2} \rightarrow B^{2}$ and plumb on the Hopf bands using Lemma 4.1.) Figure 5 is an example illustrating the following construction.

Consider a standard balls-and-link diagram in $\mathbb{R}^{3}=S^{3} \backslash\{\infty\}$ (balls for the 1-handles, a link for the 2-handles) for the given handlebody decomposition of $X$. Let $\Gamma$ be the graph in $\mathbb{R}^{2} \subset \mathbb{R}^{3}$ which is the projection of the diagram, with crossings for $L$ and balls for 1-handles made into vertices, and with dotted lines for 1-handles made into edges. By an isotopy of $L$ we can always assume $\Gamma$ is connected. Thus we have two types of vertices: 4 -valent vertices for crossings and two $(n+1)$-valent vertices for each 1-handle which has $n$ strands of the link running over it.

By plumbing left-handed Hopf bands onto a disk, one can easily construct a surface $S$ which is made up of one disk neighborhood in $\mathbb{R}^{2}$ of each vertex of $\Gamma$ and one (sometimes twisted) band neighborhood in $\mathbb{R}^{3}$ of each edge of $\Gamma$. (Start with a disk neighborhood of a spanning tree and then plumb on one Hopf band for each remaining edge.) At each 4-valent vertex corresponding to a crossing, plumb on an extra lefthanded Hopf band along an arc at right angles to one of the over-passing incident edges, underneath the surface. Now $S$ is the page of an open book decomposition of $S^{3}$ induced by a Lefschetz fibration on $B^{4}$. 


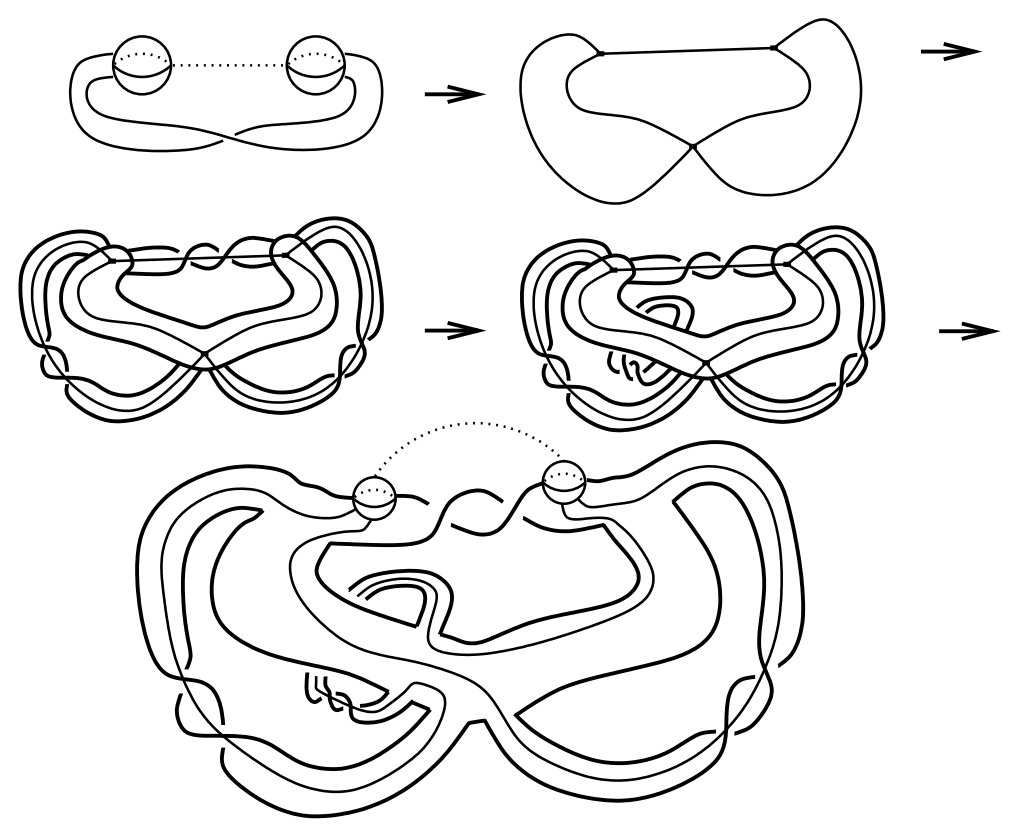

Figure 5: Constructing a Lefschetz fibration as in Proposition 4.3.

At this point, if there were no 1-handles, we would be done, since we could resolve the crossings of $\Gamma$ to reconstruct the link simply by letting the under-crossing strand at each crossing go over the extra Hopf band at that crossing. To deal with the 1-handles, at each 1-handle vertex, string the foot of the 4-dimensional 1-handle on the binding near that vertex (as in Remark 2.8) and now pass all the strands entering that vertex over the 1 -handle, remaining in the page the whole time.

To construct our BALF on the double $D X$ of a 2-handlebody $X$, we will use Proposition 4.3 on $X_{(1)}$ so that the 2 -handles lie in a page with some framing. Recall that $D X$ is constructed from $X$ by attaching, for each 2-handle of $X$, a dual 2-handle along a small linking circle with framing 0 (and then attaching 3 - and 4-handles). Thus if we slide a 2-handle from $X$ over its dual 2-handle we can change its framing by \pm 2 , so we see that the framings of the 2-handles of $X$ can be changed by \pm 2 without changing $D X$. Use this technique to change each framing to $\mathrm{pf}-1$ or $\mathrm{pf}-2$ (sliding over the dual changes the framing by \pm 2 and does not change $L$ otherwise). If $\mathrm{pf}-2$, then plumb on one more left-handed Hopf band along a short boundary-parallel arc in a page and run the attaching circle over the band so that the framing becomes $\mathrm{pf}-1$. 
Note that we have now expressed $D X$ as equal to $D X^{\prime}$ where $X^{\prime}$ has the same 0 - and 1-handles as $X$ and has 2-handles attached along the same link but with different framings than $X$. We now forget about the original $X$ and work with $X^{\prime}$, which we simply call $X$. In addition, the LF on $X_{(1)}$, with fiber $F$, in fact gives a more complicated handlebody decomposition of $X_{(1)}$, where the 1-handles are those needed to build $F \times B^{2}$, and the 2-handles are the vanishing cycles needed to turn $F \times B^{2}$ into $X_{(1)}$. We now use this, together with the rest of the 2 -handles needed to make $X$, as our handlebody decomposition of $X$, and forget the previous handlebody decomposition. Thus $D X$ is expressed as $F \times B^{2}$ together with $n 2$-handles attached along knots in a page with framing $\mathrm{pf}-1$ and $n$ more dual 2 -handles attached along small linking circles with framing 0 .

Now if we slide each dual over the 2-handle it comes from, it becomes a parallel 2 -handle, lying in a page with framing pf +1 . Thus $(D X)_{(2)}$ is expressed as a convex ALF over $B^{2}$ with $n$ Lefschetz singularities and $n$ anti-Lefschetz singularities, inducing an OBD on $\partial(D X)_{(2)}$ with trivial monodromy, since each right-handed Dehn twist has a corresponding parallel left-handed Dehn twist. (Note that at this stage we have not used any round 1 -handles.)

To finish the construction, we will construct a concave BLF on the union of the $3-$ and 4-handles of $D X$ inducing the same open book as above. The concave structure we need, after turning things upside down, is given by the following two results.

Lemma 4.4 There exists a concave BLF $f: B^{4} \rightarrow S^{2}$ which restricts to $S^{3}=\partial B^{4}$ to give the standard $O B D$ with disk pages.

Proof Take the ADK 4-sphere, discussed in Section 3 above, and remove from $S^{4}$ a 4-ball consisting of a neighborhood of a section over $D_{S}$; that is, remove the 0 -handle of each torus fiber over $D_{S}$. The result is the desired concave BLF.

(We could equally well remove the 0 -handle of each sphere fiber over $D_{S}$. However, the final BALF constructed on $D X$ will have the undesirable feature that, as we move the torus fiber over the south pole to the north pole, the genus of the fibers decreases from 1 to 0 , then increases. If we use the construction given in the proof above, however, the genus will strictly increase as we move from one pole to the other. When we finally prove Theorem 1.1, the genus will strictly increase as we move from each pole to the equator, but will not have more than one "local maximum".)

Lemma 4.5 (Attaching a 1-handle to a concave boundary) Suppose that $f: X \rightarrow \Sigma$ is a concave fibration and that $X^{\prime}$ is the result of attaching a 1 -handle to $X$. Then, after changing the handlebody decomposition of the cobordism from $\partial X$ to $\partial X^{\prime}$, we can extend $f$ to a concave fibration $f^{\prime}: X^{\prime} \rightarrow \Sigma$ with the following properties. 
(1) Each page of the new $O B D$ on $\partial X^{\prime}$ is diffeomorphic to a page of the $O B D$ on $\partial X$ with a 2-dimensional 1-handle attached along two intervals in the binding. (The locations of these intervals can be chosen in advance.)

(2) The monodromy of the new $O B D$ is the monodromy of the old $O B D$ extended by the identity across the 2-dimensional 1-handle.

(3) The only singularity in $f^{\prime}: X^{\prime} \rightarrow \Sigma$ that is not in $f: X \rightarrow \Sigma$ is a single round 1-handle singularity.

Proof Let $I_{0}$ and $I_{1}$ be the two intervals in the binding along which the 2-dimensional 1 -handle is to be attached. Move one foot of the 4-dimensional 1-handle into a ball neighborhood $B_{0}$ of $I_{0}$ and the other into a ball neighborhood $B_{1}$ of $I_{1}$. Inside $B_{0}$ introduce a cancelling 2-3-handle pair so that the 2-handle is attached along a 0 -framed unknot $K$ and the 3-handle is attached along a 2 -sphere $S$ made of the Seifert disk for $K$ and the core disk of the 2-handle. Now slide an arc of $K$ over the 1-handle so that we see one unknotted loop of $K$ sticking out of the 1-handle in the ball $B_{0}$ and another unknotted loop sticking out of the 1-handle in the ball $B_{1}$. Now push each loop across the binding, and the 1-handle together with the 2-handle becomes a round 1-handle as in Remark 2.5, across which the fibration $f$ extends. This much is illustrated in Figure 6.

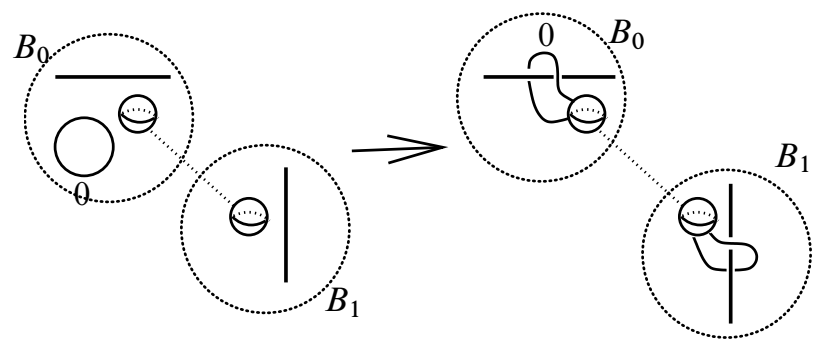

Figure 6: Attaching a 1-handle to a concave boundary.

Now observe that the page has changed by removing a disk near $I_{0}$ and a disk near $I_{1}$ and replacing with $[0,1] \times S^{1}$, with the monodromy extended by the identity across $[0,1] \times S^{1}$, as illustrated in Figure 7 .

The 3-handle can then be seen to be attached along the 2-sphere which intersects each page in the arc $A$ drawn in Figure 7. Thus the fibration extends across the 3-handle as in Remark 2.8. The page has now changed by cutting open along $A$, which amounts to attaching a 2-dimensional 1-handle to the original page of $f$ at the two intervals $I_{0}$ and $I_{1}$. 


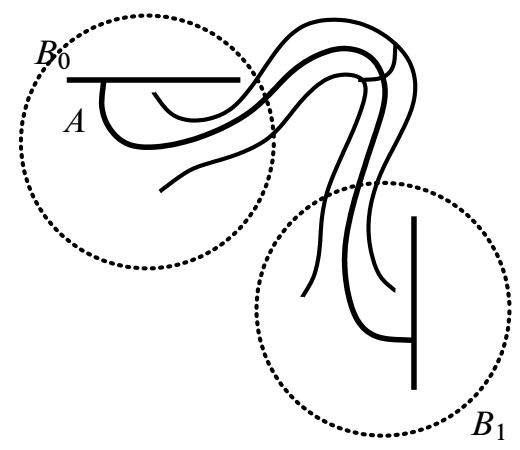

Figure 7: How the page changes after attaching a 1-handle to a concave boundary.

Using these two lemmas, build a concave BLF on the union of the 3- and the 4handles which has an OBD on its boundary with trivial monodromy and with pages diffeomorphic to the pages coming from the convex BLF on $(D X)_{(2)}$. This can be done because the number of 3-handles in $D X$ equals the number of 1-handles in $D X$ which equals the number of 1 -handles in each page on $\partial(D X)_{(2)}$; also Lemma 4.5 gives us the freedom to attach the 2-dimensional 1-handles to the pages so as to get the right number of boundary components.

Now glue the two pieces together using the diffeomorphism we get by identifying their open books. This gives $X$ for the following reason: The 4 and 3-handles of $X$ form a boundary connected sum of $S^{1} \times B^{3}$ s. By a classical theorem of Laudenbach and Poenaru [26] it does not matter which diffeomorphism of a connected sum of $S^{1} \times S^{2}$ 's is used to glue on the 4-and 3-handles; the resulting 4-manifolds are diffeomorphic. Thus, in gluing trivial open book to trivial open book above, we must obtain $X$.

Thus we have proved the following proposition.

Proposition 4.6 If $X^{4}$ is a 2-handlebody then its double $D X$ has a BALF $f: D X \rightarrow$ $S^{2}$.

\subsection{The Andrews-Curtis conjecture}

One way of constructing smooth, homotopy 4-spheres which may not be diffeomorphic to $S^{4}$ (they are homeomorphic, see Freedman [14]) is to use balanced presentations of the trivial group which are not known to satisfy the Andrews-Curtis Conjecture.

If a finite presentation of a group $G$ is described by attaching 1- and 2-handles to an $n$-ball, then sliding handles over handles and introducing or cancelling 1-2-handle 
pairs correspond to what are called Andrews-Curtis moves on the presentation. The Andrews-Curtis conjecture is that any balanced presentation of the trivial group can be reduced to the trivial presentation using only these moves. The point is that you "can't remember", meaning that at any moment the only relations available for use are those of current 2-handles. (When one 2-handle slides over another the old relation represented by the old $2-$ handle is lost.)

A balanced presentation $P=\left\{x_{1}, \ldots, x_{n} \| r_{1}, \ldots, r_{n}\right\}$ of the trivial group determines uniquely a homotopy 4 -sphere by attaching $n 1$-handles to the 5-ball, and then $n$ 2-handles whose attaching maps read off the relations $\left\{r_{1}, \ldots, r_{n}\right\}$. If two attaching maps represent the same relation, then they are homotopic, and homotopic circles in dimension 4 are isotopic. Hence this 5 -manifold $V^{5}$ is unique up to diffeomorphism and is contractible. Its boundary $\partial V=S_{P}$, is the homotopy 4-sphere associated with the presentation $P$.

Given $P$, we can also build 4-manifolds $X$ which are contractible by adding $n 1-$ handles to the 4-ball and then $n$ 2-handles corresponding to the relations. This involves choices because different attaching maps which are homotopic are not necessarily isotopic, so there are many possible choices of $X$ corresponding to $P$. However in all cases, $X \times I$ is diffeomorphic to $V^{5}$ because with the extra dimension homotopic attaching maps are isotopic. We have shown the following lemma.

Lemma 4.7 Our homotopy 4-sphere $\partial V$ is diffeomorphic to $\partial(X \times I)$ and hence diffeomorphic to the double $D X$ which is known to be a BALF.

Question 4.8 Is the fact that $\partial V$ is known to be a BALF helpful in showing that $\partial V$ is, or is not, diffeomorphic to $S^{4}$ ?

Remark 4.9 If a presentation $P$ can be reduced to the trivial presentation by AndrewsCurtis moves, then these moves can be mirrored geometrically in handle slides, and then $V^{5}=B^{5}$ so $D X$ is $S^{4}$.

But it is possible that $D X$ is diffeomorphic to $S^{4}$ even though $P$ cannot be reduced to the trivial presentation by Andrews-Curtis moves. This would have to be the case if the Andrews-Curtis Conjecture is false (as is expected by many experts) and the smooth 4-dimensional Poincaré Conjecture is true.

The authors know of only one presentation $P$, namely $\left\{x, y \| x y x=y x y, x^{4}=y^{5}\right\}$, which is not known to satisfy the Andrews-Curtis Conjecture but is known to give $S^{4}$. The latter was shown in Akbulut and Kirby $[1 ; 2]$ with a beautiful denouement by Gompf in [18].

There are many tantalizing presentations to play with. A full discussion appears in Hog-Angeloni and Metzler [23]. 


\section{The general construction of convex 2-handlebodies}

To prove Theorem 1.1 we will need a general construction of convex BLFs on 2handlebodies, with prescribed boundary conditions. As a warm-up we prove a simple version without the boundary conditions.

Proposition 5.1 (Quick and easy recipe for convex BLFs) Every 4-dimensional 2-handlebody $X$ can be given the structure of a convex BLF.

Proof Let $f: X_{(1)} \rightarrow D^{2}$ be the LF whose existence is asserted by Proposition 4.3. The idea now is to turn each 2-handle (whose attaching circle lies in a page of the open book on $\left.\partial X_{(1)}\right)$ into a round 1-handle whose attaching circles are transverse to the pages of the open book. For each such attaching circle $K$ of a 2 -handle $H$, consider a neighborhood $U$ of the arc $A$ mentioned in Proposition 4.3, in which we see only an arc of the binding $B$ and an arc of $K$ lying in a half-disk of the page $F$. The following construction is illustrated in Figure 8.

First introduce a cancelling 1-2-handle pair inside $U$ so that the feet of the 1-handle intersect $F$ in small disks, so that the attaching circle of the cancelling 2-handle runs from one foot straight to the other staying in $F$ with framing -1 with respect to this picture. Next, slide a small loop of $K$ over the 1 -handle, and now $H$ together with the 1-handle form a round 1-handle $H^{\prime}$; the two attaching circles of $H^{\prime}$ are a small unknot $U$ near $B$ and a copy $K^{\prime}$ of the original knot $K$. Now push $U$ across $B$ to become a small meridinal loop, hence transverse to the pages of the open book. Likewise, push a small finger out from $K^{\prime}$ and across $B$ and then tilt the rest of $K^{\prime}$ out of the page $F$ so that $K^{\prime}$ also becomes transverse to the pages. Thus the two feet of this round 1-handle wrap once around the binding and the broken Lefschetz fibration extends across the round 1-handle. Lastly note that the cancelling -1 -framed 2-handle now lies in the extended page (after attaching the round 1-handle) and has framing $\mathrm{pf}-1$, so the fibration also extends across these 2 -handles.

For the more general result we need to keep track of almost complex structures and homotopy classes of plane fields associated to fibrations and OBDs.

Given a $\mathrm{B}(\mathrm{A}) \mathrm{LF} f: X \rightarrow \Sigma$, we will use $R_{f}$ to denote the union of the round 1handle singularities. Given a 4-manifold $X$, let $\mathcal{J}(X)$ be the set of all almost complex structures on $X$ modulo homotopy. Given a 3-manifold $M$ let $\mathcal{Z}(M)$ be the set of all co-oriented plane fields on $M$ modulo homotopy. (This is of course equivalent to the set of all nowhere-zero vector fields modulo homotopy, but we take the plane field perspective because of the connections with contact topology.) First note the following facts relating Lefschetz fibrations, almost complex structures, open book decompositions and homotopy classes of plane fields. 


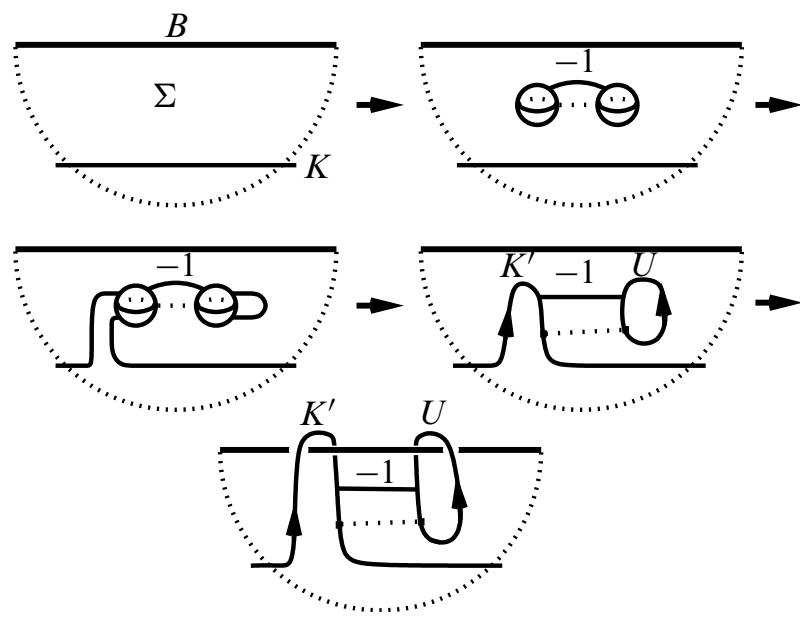

Figure 8: Turning a 2-handle into a round 1-handle (proof of Proposition 5.1).

(1) A BLF $f$ on a 4-manifold $X$ determines a homotopy class $j(f) \in \mathcal{J}\left(X \backslash R_{f}\right)$, characterized by having a representative $J \in j(f)$ such that the fibers of $f$ are $J$-holomorphic curves.

(2) An OBD $f$ on a 3-manifold $M$ determines a homotopy class $z(f) \in \mathcal{Z}(M)$, characterized by having a representative which is positively transverse to a vector field $V$ which in turn is positively transverse to the pages of $f$ and positively tangent to the binding of $f$. This is the same as the homotopy class of the unique isotopy class of positive contact structures supported by $f$ in the sense of Giroux [17].

(3) If $X$ is a 4-manifold and $M=\partial X$, then a homotopy class $j \in \mathcal{J}(X)$ determines a homotopy class $z(j) \in \mathcal{Z}(M)$ characterized by having a representative $\xi$ which is the field of $J$-complex tangencies to $M$ for some $J \in j$.

(4) If $f$ is a convex BLF on a 4-manifold $X$, inducing the OBD $\left.f\right|_{M}$ on $M=\partial X$, then $z(j(f))=z\left(\left.f\right|_{M}\right)$.

Theorem 5.2 Let $X$ be a 4-dimensional 2-handlebody, let $C$ be a nonempty finite disjoint union of points and circles in the interior of $X$ and let $J$ be an almost complex structure on $X \backslash C$. Let $N$ be a given open neighborhood of $C$. Then there exists a convex BLF $f: X \rightarrow B^{2}$ with the following properties.

- The union of the round 1-handle singularities $R_{f}$ is contained in $N$.

- For any almost complex structure $J^{\prime} \in j(f), J$ and $J^{\prime}$ will be homotopic on $X \backslash N$. 
- The positive contact structure supported by $\left.f\right|_{\partial X}$ is overtwisted.

At this point it is worth emphasizing that, to prove Theorem 1.1, we would be satisfied if Theorem 5.2 produced a BALF. However, we feel it is of independent interest that we are able to avoid achirality on the convex half of the construction. Before we prove Theorem 5.2, the corollary that we will actually use is the following.

Corollary 5.3 Given any 4-dimensional 2-handlebody $X$ and any OBD $g: \partial X \rightarrow$ $B^{2}$ which supports an overtwisted contact structure $\xi$, there exists a convex $B L F$ $f: X \rightarrow B^{2}$ such that the open book $\left.f\right|_{\partial X}$ is obtained from $g$ by a sequence of positive stabilizations.

Proof The homotopy class of plane fields $z(g)$ on $\partial X$ determines a homotopy class of almost complex structures on a collar neighborhood of $\partial X$, which extends across all of $X$ except perhaps a finite disjoint union of points and circles. (This is because the space of almost complex structures on $\mathbb{R}^{4}$ respecting a given metric is $S^{2}$, so we only see obstructions to extending almost complex structures when we reach the $3-$ skeleton.) Then Theorem 5.2 produces a BLF $f^{\prime}$, such that $\xi \in z\left(\left.f^{\prime}\right|_{\partial X}\right)$. Eliashberg's classification of overtwisted contact structures [11] tells us that the contact structure supported by $\left.f^{\prime}\right|_{\partial X}$ is isotopic to $\xi$, and Giroux's results on contact structures and open books then tell us that $\left.f^{\prime}\right|_{\partial X}$ and $g$ have a common positive stabilization $h: \partial X \rightarrow B^{2}$ (where stabilization is plumbing on left-handed Hopf bands). Lastly each stabilization of $\left.f^{\prime}\right|_{\partial X}$ required to produce $h$ can be implemented using Lemma 4.1, to produce a BLF $f: X \rightarrow B^{2}$ such that $\left.f\right|_{\partial X}=h$.

To prove Theorem 5.2 (producing a BLF rather than a BALF) we need a way of negatively stabilizing OBDs on convex boundaries without introducing anti-Lefschetz singularities. Figure 9 shows a modification of an OBD involving plumbing one righthanded Hopf band along an arc $A$ in a page, one left-handed Hopf band along a parallel copy of $A$, and one more left-handed Hopf band along a short arc transverse to this parallel copy. We will now show that this modification can be achieved using round 1-handles but no anti-Lefschetz singularities. One should think of the following lemma as giving us the freedom to plumb on right-handed Hopf bands wherever we want, avoiding achirality, at the expense of introducing extraneous left-handed Hopf bands.

Lemma 5.4 Given a convex (BA)LF $f: X \rightarrow B^{2}$ and an arc $A$ in a page of the $O B D$ on $\partial X$, there exists a $B(A) L F f^{\prime}: X \rightarrow B^{2}$ inducing the $O B D$ indicated in Figure 9, which agrees with $f$ outside a neighborhood $U$ of $A$ and which has one Lefschetz singularity and one round 1 -handle singularity inside $U$. 


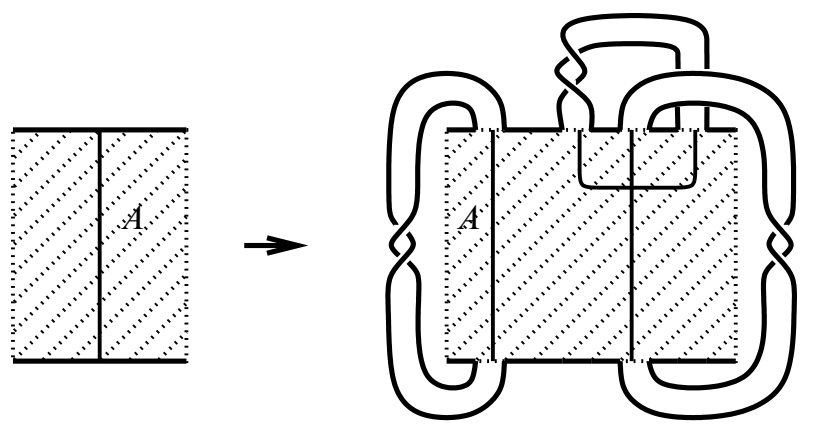

Figure 9: Plumbing on one right-handed Hopf band along an arc $A$, together with a left-handed Hopf band plumbed along a parallel copy of $A$ and a left-handed Hopf band plumbed along a short arc transverse to this parallel copy.

Proof Attach two cancelling 1-2-handle pairs as on the left in Figure 10 (so we have not changed the 4-manifold). Then observe, as on the right in Figure 10, that this configuration can also be seen as a 1-handle with feet strung on the binding, a round 1-handle with feet wrapping once around the binding and a 2-handle whose foot is a knot in a page running over the round 1-handle, with framing $\mathrm{pf}-1$. The monodromy of the new open book decomposition is indicated on the left in Figure 11. To see this, note that we would like to see both feet of the round 1-handle as given by fixed points of the monodromy, but the left foot goes over the 1-handle. However, if we introduce a left-handed Dehn twist and a right-handed Dehn twist along parallel curves that go along the arc $A$ and over the 1-handle (the product of which is isotopic to the identity), the section determined by a fixed point in between the two twists is in fact the same as the left foot of the round 1-handle. The extra right-handed Dehn twist in Figure 11 comes from the -1 framed vanishing cycle 2-handle. Figure 11 then shows a two-step isotopy so that we see that the resulting monodromy agrees with the monodromy for Figure 9. (In these figures the indicated monodromy should be understood to be composed with any pre-existing monodromy coming from the initial open book decomposition.) Thus the new page is isotopic to that in Figure 9. (To go from a statement about the monodromy of an open book to a statement about the isotopy class of an open book is not safe in general. Here, however, we have the fact that the operation in question amounts to a Murasugi sum with an open book decomposition of $S^{3}$, and in $S^{3}$ open book decompositions are completely determined up to isotopy by their monodromy, since the mapping class group of $S^{3}$ is trivial.) 

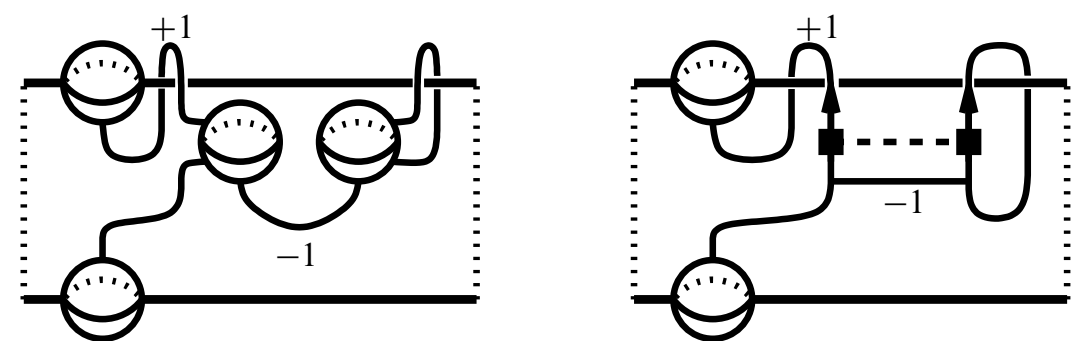

Figure 10: Two cancelling 1-2-handle pairs becoming a 1-handle, a round 1-handle and a 2-handle.
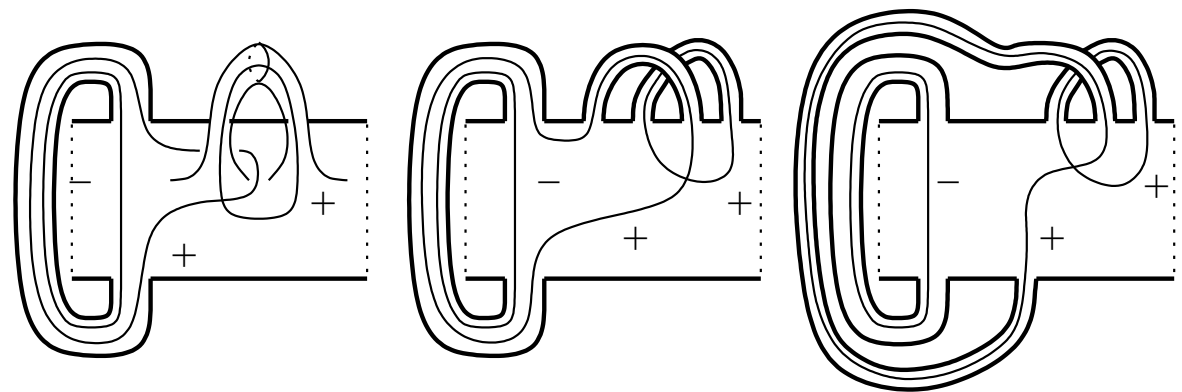

Figure 11: Three equivalent descriptions of the monodromy corresponding to Figure 10.

The last techniques we need to develop before the proof of Theorem 5.2 are techniques for computing Chern classes of almost complex structures and invariants of co-oriented plane fields in terms of BLFs and OBDs. We begin by collecting some relevant facts.

First, note that there is a well-defined connected sum operation \# on $\left\{\left(M^{3}, z\right) \mid z \in\right.$ $\mathcal{Z}(M)\}$, since any two plane fields are locally homotopic. Likewise there is a welldefined boundary connected sum operation $\natural$ on $\left\{\left(X^{4}, j\right) \mid j \in \mathcal{J}(X)\right\}$ which induces the connected sum on the boundary. (These extend in the obvious way to self-connect sums and boundary self-connect sums.) If one attaches a 1-handle from one convex BLF $\left(X_{1}, f_{1}\right)$ to another one $\left(X_{2}, f_{2}\right)$ such that the feet are strung on the bindings as in Remark 2.8, giving a BLF $f$ on $X_{1} \natural X_{2}$, the resulting $j(f) \in \mathcal{J}\left(\left(X_{1} \curvearrowleft X_{2}\right) \backslash R_{f}\right)$ is equal to the boundary connect sum $j\left(f_{1}\right) \downarrow j\left(f_{2}\right)$, and $z(f) \in \mathcal{Z}\left(\partial X_{1} \sharp \partial X_{2}\right)$ is equal to $z\left(f_{1}\right) \sharp z\left(f_{2}\right)$.

Next, we summarize some results from [19]; a useful exposition can also be found in Ding, Geiges and Stipsicz [8]. 
There are two invariants $d_{2}$ and $d_{3}$ of $\mathcal{Z}(M)$, which as a pair constitute a complete invariant. The "2-dimensional invariant" $d_{2}$ of a given $z \in \mathcal{Z}(M)$ is simply the $\operatorname{spin}^{\mathbb{C}}$ structure determined by $z$; in the case where $H^{2}(M ; \mathbb{Z})$ has no 2 -torsion, this is completely characterized by $c_{1}(z) \in H^{2}(M ; \mathbb{Z})$. In general, the set $\mathcal{S}(M)$ of $\operatorname{spin}^{\mathbb{C}}$ structures on $M$ is an affine space for $H^{2}(M ; \mathbb{Z})$, and the action of $H^{2}(M ; \mathbb{Z})$ on $\mathcal{S}(M)$ has the property that $c_{1}(a \cdot s)=2 a+c_{1}(s)$ for $a \in H^{2}(M ; \mathbb{Z})$ and $s \in \mathcal{S}(M)$. The "3-dimensional invariant" $d_{3}(z)$ lies in an affine space for a cyclic group; the key properties of $d_{3}$ that we need are summarized in the following two items.

Focusing on the case of $M=S^{3}$ (in which case there is only one $\operatorname{spin}^{\mathbb{C}}$ structure and so we need only pay attention to $\left.d_{3}\right)$ suppose that $z_{1}, z_{2} \in \mathcal{Z}\left(S^{3}\right)$ and that $S^{3}=\partial X_{1}=\partial X_{2}$, with $j_{i} \in \mathcal{J}\left(X_{i}\right)$ such that $\left.j_{i}\right|_{S^{3}}=z_{i}$, for $i=1,2$. Then $c_{1}\left(j_{1}\right)^{2}-$ $2 \chi\left(X_{1}\right)-3 \sigma\left(X_{1}\right)=c_{1}\left(j_{2}\right)^{2}-2 \chi\left(X_{2}\right)-3 \sigma\left(X_{2}\right)$ if and only if $d_{3}\left(z_{1}\right)=d_{3}\left(z_{2}\right)$ (ie if and only if $\left.z_{1}=z_{2}\right)$. Hence, in the case of $S^{3}$, we identify $d_{3}(z)$ with $\left(c_{1}(j)^{2}-2 \chi(X)-\right.$ $3 \sigma(X)) / 4 \in \mathbb{Z}-1 / 2$, where $j$ is any extension of $z$ over a 4 -manifold $X$. Now, for a general 3-manifold $M$, if $z_{1}, z_{2} \in \mathcal{Z}(M)$ and $d_{2}\left(z_{1}\right)=d_{2}\left(z_{2}\right)$, then there exists a $z \in \mathcal{Z}\left(S^{3}\right)$ such that $d_{3}\left(z_{2}\right)=d_{3}\left(z_{1} \# z\right)$. In particular, $\left(M, z_{2}\right)=\left(M, z_{1}\right) \#\left(S^{3}, z\right)$. If $M=S^{3}$, then $d_{3}\left(z_{2}\right)=d_{3}\left(z_{1}\right)+d_{3}(z)+1 / 2$.

Now we summarize a discussion in Gay and Kirby [16] on constructing almost complex structures in prescribed homotopy classes.

Given an almost complex structure $J$ on a smooth manifold $X, J$ can always be trivialized over the 1-skeleton of $X$. Then $c_{1}(J)$ is represented by the cocycle whose value on a 2-cell $e$ is the obstruction to extending this trivialization across $e$, as an element of $\pi_{1}\left(G L_{2}(\mathbb{C})\right)=\mathbb{Z}$. Any two almost complex structures can be made, via a homotopy, to agree on the 1 -skeleton. Given two almost complex structures $J_{1}$ and $J_{2}$ over the 2-skeleton which agree on the 1-skeleton, if their obstruction cocycles are equal for a given trivialization over the 1 -skeleton then $J_{1}$ is homotopic to $J_{2}$ on all of the 2-skeleton. Thus, if we wish to construct a given almost complex structure up to homotopy on a 2-handlebody, we must be able to construct an almost complex structure $J_{1}$ on the $1-$ skeleton with a trivialization and then, for any given cocycle $c$, be able to extend $J_{1}$ to an almost complex structure $J$ on the 2 -skeleton with $c$ as its obstruction cocycle. In the absence of 2 -torsion in $H^{2}(X ; \mathbb{Z})$, this just amounts to getting $c_{1}(J)$ correct, but when there is 2 -torsion, there will be different cocycles representing a fixed $c_{1}$ but corresponding to different almost complex structures.

Next we combine some standard contact and symplectic topology and some results from [8] to relate the above facts to surgery and handle addition:

Given a 3-manifold $M$ and a homotopy class $z \in \mathcal{Z}(M)$, suppose that $\xi \in z$ and that $K$ is a knot in $M$ tangent to $\xi$. Then $K$ comes with a canonical framing $c$ given by $\xi$; 
let $M^{\prime}$ be the result of $c \pm 1$ surgery on $K$. Then there is a well-defined $z^{\prime} \in \mathcal{Z}\left(M^{\prime}\right)$ which can be characterized in either of the following two equivalent ways.

(1) Homotope $\xi$, remaining fixed along $K$, to be positive contact in a neighborhood of $K$. Then there is a unique contact \pm 1 surgery along $K$, producing $\xi^{\prime}$ on $M^{\prime}$, and we let $z^{\prime}$ be the homotopy class of $\xi^{\prime}$.

(2) Express a neighborhood $N$ of $K$ as $S^{1} \times[-1,1] \times[-1,1]$, with $K=S^{1} \times 0 \times 0$ and with $\xi$ tangent to $S^{1} \times[-1,1] \times 0$ along $K$. Now homotope $\xi$, remaining fixed along $K$, to be tangent to the foliation $S^{1} \times[-1,1] \times t$ on all of $N$. As in Lickorish [27], $c \pm 1$ surgery along $K$ can be viewed as cutting $M$ open along $S^{1} \times[-1,1] \times 0$ and reglueing via a left/right-handed Dehn twist along $K$. Thus the surgered neighborhood $N^{\prime}$ in $M^{\prime}$ naturally inherits a foliation by annuli, and we let $\xi^{\prime}$ be tangent to this foliation inside $N^{\prime}$ and be equal to $\xi$ outside the surgery. Then we define $z^{\prime}$ to be the homotopy class of $\xi^{\prime}$.

Now suppose that $X$ is a 4-manifold with $\partial X=M$ and with a given $j \in \mathcal{J}(M)$ restricting to $z \in \mathcal{Z}(M)$. Let $\xi \in z$ with $K$ tangent to $\xi$ as above, with canonical framing $c$. Let $X^{\prime}$ be the result of attaching a 2-handle $H$ along $K$ with framing $c \pm 1$, so that $\partial X^{\prime}=M^{\prime}$ as above, and let $z^{\prime} \in \mathcal{Z}\left(M^{\prime}\right)$ be as above. Then, in the case of $c-1$ framing, there is a canonical extension $j^{\prime}$ of $j$ across $H$ so that $\left.j^{\prime}\right|_{M^{\prime}}=z^{\prime}$, and in the case of $c+1$ framing, there is a canonical extension $j^{\prime}$ of $j$ across $H \backslash B$, where $B$ is a small ball in the interior of $H$, so that $\left.j^{\prime}\right|_{M^{\prime}}=z^{\prime}$. These extensions can be characterized as follows.

(1) In the case of $c-1$ framing, identify $H=D^{2} \times D^{2}$ as a subset of $\mathbb{C}^{2}$ via the orientation-preserving map $D^{2} \times D^{2} \ni\left(\left(x_{1}, x_{2}\right),\left(y_{1}, y_{2}\right)\right) \mapsto\left(x_{1}+i y_{1}, x_{2}-\right.$ $\left.i y_{2}\right)=\left(z_{1}, z_{2}\right) \in \mathbb{C}^{2}$. Then $j^{\prime}$ is represented by an almost complex structure $J^{\prime} \in j^{\prime}$ which equals the standard integrable complex structure on $H \subset \mathbb{C}^{2}$ and, when restricted to $X=X^{\prime} \backslash H$, represents $j$. In particular, the fibers of the map $\left(z_{1}, z_{2}\right) \mapsto z_{1}^{2}+z_{2}^{2}$ in $H \subset \mathbb{C}^{2}$ are $J^{\prime}$-holomorphic.

(2) In the case of $c+1$ framing, identify $H=D^{2} \times D^{2}$ as a subset of $\mathbb{C}^{2}$ via the orientation-reversing map $D^{2} \times D^{2} \ni\left(\left(x_{1}, x_{2}\right),\left(y_{1}, y_{2}\right)\right) \mapsto\left(x_{1}+i y_{1}, x_{2}+\right.$ $\left.i y_{2}\right)=\left(z_{1}, z_{2}\right) \in \mathbb{C}^{2}$. Then $j^{\prime}$ is represented by an almost complex structure $J^{\prime} \in j^{\prime}$ defined everywhere except at $(0,0) \in H$ which, when restricted to $X=X^{\prime} \backslash H$, represents $j$ and which is characterized on $H$ by the fact that the fibers of the map $\left(z_{1}, z_{2}\right) \mapsto z_{1}^{2}+z_{2}^{2}$ are $J^{\prime}$-holomorphic except at $(0,0)$. The ball $B$ is then a small ball around $(0,0)$. Although $j^{\prime}$ does not extend across $B$, if we replace $B$ with $\mathbb{C} P^{2} \backslash B^{4}$ (ie connect sum with $\mathbb{C} P^{2}$ ), then $j^{\prime}$ does extend across $\mathbb{C} P^{2} \backslash B^{4}$ so as to agree with the standard complex structure on $\mathbb{C} P^{2}$. 
Now suppose that, in the setting of the preceding paragraph, we are also given a trivialization of $\xi$ in a neighborhood of $K$ (ie a non-vanishing section $v$ of $\xi$ ). This gives $K$ a rotation number $\operatorname{rot}(K)$ (the winding number of $T K$ inside $\xi$ relative to the trivialization). Suppose that $J \in j$ so that $\xi$ is the field of $J$-complex tangencies to $M$; then we naturally get a trivialization $(v, n)$ of $J$ in a neighborhood of $K$, where $n$ is the outward normal to $M$. Let $J^{\prime} \in j^{\prime}$ agree with $J$ on $X$. Then, in both the case of $c-1$ framing and $c+1$ framing, the obstruction to extending this trivialization of $J$ to a trivialization of $J^{\prime}$, as an element of $\pi_{1}\left(G L_{2}(\mathbb{C})\right)=\mathbb{Z}$, is precisely $\operatorname{rot}(K)$. (In [8] this is proved in the case where $X=B^{4}, \xi$ is the standard contact structure on $S^{3}, v$ is defined on all of $S^{3}$, and $c \neq 0$. Note, however, that our assertion is purely local to $K$ and $H$, and that, given any $\xi$ on $S^{1} \times B^{2}$ which is tangent to $S^{1} \times\{0\}$, with any trivialization $v$ of $\xi$, after a homotopy of $\xi$ fixed along $K$ there exists an embedding of $S^{1} \times B^{2}$ into $S^{3}$ carrying $\xi$ to the standard contact structure on $S^{3}$, taking $S^{1} \times\{0\}$ to a Legendrian knot with tb $\neq 0$, and taking $v$ to a trivialization which extends over all of $S^{3}$.)

Finally, if $X$ is equipped with a convex (BA)LF $f: X \rightarrow B^{2}$ and if $\left(X^{\prime}, f^{\prime}\right)$ is the (BA)LF resulting from attaching a 2 -handle along a knot in a page of the induced OBD on $\partial X$ with framing $\operatorname{pf} \pm 1$, then $j\left(f^{\prime}\right)=j(f)^{\prime}$ in the sense that $j\left(f^{\prime}\right)$ is precisely the canonical extension of $j(f)$ discussed above.

This gives us the following algorithm for computing the invariants of a homotopy class $z \in \mathcal{Z}(M)$ associated to an open book decomposition on a closed 3-manifold $M$ in terms of a factorization $h=\tau_{1} \circ \cdots \circ \tau_{n}$ of the monodromy $h$ into Dehn twists $\tau_{i}$ along curves $\gamma_{i}$ in the page $F$. (We hope some readers may find this algorithm useful in other contexts; a similar algorithm is spelled out in [13].)

(1) Begin with a standard immersion of the page $F$ in $\mathbb{R}^{2}$ as a disk with 2dimensional 1-handles attached around the boundary.

(2) This gives a trivialization of $T F$ coming from the standard trivialization of $T \mathbb{R}^{2}$. Together with the standard trivialization of $T B^{2}$, we get a trivialization of $T\left(F \times B^{2}\right)$ which yields a trivialization of the standard almost complex structure on $F \times B^{2}$.

(3) Each Dehn twist curve $\gamma_{i}$ can be thought of as a curve in $F \times p_{i}$, where $p_{i} \in S^{1}$; with respect to the above trivialization, we get a rotation number $\operatorname{rot}\left(\gamma_{i}\right)$ which is precisely the winding number of $\gamma_{i}$ as an immersed curve in $\mathbb{R}^{2}$, seen via the immersion of $F$ in $\mathbb{R}^{2}$.

(4) Now interpret the Dehn twist curves as attaching circles for 2-handles attached to $F \times D^{2}$, with framing pf -1 for each right-handed Dehn twist and framing pf +1 for each left-handed Dehn twist. This describes an ALF on a 4-manifold 
$X$ with an almost complex structure $J$ on the complement of $q$ points, where $q$ is the number of left-handed Dehn twists, and $\left.J\right|_{\partial X}$ induces the required homotopy class $z$ of plane fields on $M=\partial X$.

(5) Then $J$ extends to an almost complex structure $J^{\prime}$ on all of $X^{\prime}=X \sharp^{q} \mathbb{C} P^{2}$ which is standard on each $\mathbb{C} P^{2}$ summand, and we still have $\left.J^{\prime}\right|_{\partial X^{\prime}}=\left.J\right|_{\partial X}$ inducing $z$ on $M=\partial X^{\prime}$.

(6) Now read off $c_{1}\left(J^{\prime}\right)$ as a cocycle from the rotation numbers of each $\gamma_{i}$ and the fact that $c_{1}$ evaluates to 3 on each generator of $H_{2}\left(X^{\prime}\right)$ coming from a $\mathbb{C} P^{2}$ summand.

(7) Now use the intersection form on $X^{\prime}$ to identify the Poincare dual of $c_{1}\left(J^{\prime}\right)$ and hence compute $\left.c_{1}\left(J^{\prime}\right)\right|_{\partial X^{\prime}}$ to get $d_{2}(z)$ and compute $\chi\left(X^{\prime}\right), \sigma\left(X^{\prime}\right)$ and $c_{1}\left(J^{\prime}\right)^{2}$ to get $d_{3}(z)=\left(c_{1}\left(J^{\prime}\right)^{2}-2 \chi\left(X^{\prime}\right)-3 \sigma\left(X^{\prime}\right)\right) / 4$.

(8) The last two steps are equivalent to the following shortcut: Read off $c_{1}(J)$ from the rotation numbers of each $\gamma_{i}$. Use the intersection form on $X$ to identify $c_{1}(J)$ and $\left.c_{1}(J)\right|_{\partial X}$ to get $d_{2}(z)$. Then compute $\chi(X), \sigma(X)$ and $c_{1}(J)^{2}$ to get $d_{3}(z)=\left(c_{1}(J)^{2}-2 \chi(X)-3 \sigma(X)\right) / 4+q$.

Proof of Theorem 5.2 First we will prove the theorem when $X=B^{4}$ and $C$ is a point. Then we will prove it when $X=S^{1} \times B^{3}$ and $C=S^{1} \times\{0\}$. Finally we will prove the general case.

Simplest case: $X=B^{4}$ and $C$ is a single point In this case all we need to do is to construct a broken Lefschetz fibration on $B^{4}$ inducing a given homotopy class of plane fields on $S^{3}$. Recall that $\mathcal{Z}\left(S^{3}\right)$ is in one-to-one correspondence with $\mathbb{Z}-1 / 2$ via the formula $d_{3}(z)=\left(c_{1}(j)^{2}-2 \chi(X)-3 \sigma(X)\right) / 4$, where $j$ is an extension of $z$ over a 4-manifold $X$. Thus, suppose we are given $n \in \mathbb{Z}$, and we wish to construct a convex BLF on $B^{4}$ inducing a given $z \in \mathcal{Z}\left(S^{3}\right)$ with $d_{3}(z)=n-1 / 2$.

It is well known that plumbing on a left-handed Hopf band will not change $d_{3}$ (in fact it does not change the isotopy class of the contact structure $[17 ; 35]$ ), while plumbing on a right-handed Hopf band increases $d_{3}$ by one [35]. Furthermore the trivial fibration $B^{4}=B^{2} \times B^{2} \rightarrow B^{2}$ yields $d_{3}=-1 / 2$. Thus, using Lemma 5.4 we can achieve our goal for any $n>0$ (getting overtwistedness from the fact that we have plumbed on at least one right-handed Hopf band). By the comments on connected sums above, we now need only perform the construction for some negative value of $n$ to complete the proof when $X=B^{4}$. (If we want $d_{3}(z)=n-1 / 2$ we should first target $d_{3}(z)=n-1-1 / 2$ and then plumb on an extra right-handed Hopf band using Lemma 5.4 to get overtwistedness and $d_{3}(z)=n-1 / 2$.) 
We give a construction explicitly in Figure 12 for $n=-1$, ie $d_{3}=-3 / 2$; the figure should be interpreted as follows: The topmost diagram shows a page of an open book decomposition of $S^{3}$ involving 2 left-handed Hopf bands and 2 right-handed Hopf bands plumbed in sequence onto a disk, so that the page is a 4 -punctured disk. Each right-handed Hopf band should really have an extra pair of left-handed Hopf bands immediately adjacent, as in Figure 9, but we have suppressed this extra pair since they play no further role in the construction. This open book decomposition of $S^{3}$ (including the 4 extra left-handed Hopf bands not drawn) is thus the boundary of a convex BLF, using Lemma 5.4 and Lemma 4.1. To this we add a 1-handle strung along the binding, a round 1-handle which wraps around the binding once, and a 2-handle on a page with framing pf -1 running over the round 1 -handle, as in the Figure. This gives a more complicated convex BLF on $B^{4}$.

To see that we have achieved $n=-1$, we first analyze the monodromy of the new open book decomposition of $S^{3}$, exactly as in the proof of Lemma 5.4, Figure 11. We need to introduce pairs of right- and left-handed Dehn twists parallel to and on either side of the two feet of the round 1-handle to compensate for the fact that the feet are not initially described as fixed points of the monodromy. This is indicated in the middle diagram in Figure 12. The Dehn twist curves are labelled and oriented for use in the calculation to come. We now use this factorization of the monodromy into Dehn twists to compute $d_{3}$ as in the algorithm explained above. This describes a new 4-manifold shown in the bottom diagram in the figure; each right- (resp. left-) handed Dehn twist has become a $2-$ handle on a page with framing -1 (resp. +1$)$, attached to an open book with page a 6 -punctured torus and monodromy equal to the identity. We note that $H_{2}$ is generated by $A=c-a_{1}-b_{1}-a_{2}-b_{2}$ and $B=d+f-g-a_{1}-b_{1}-a_{2}-b_{2}$, with $A^{2}=1$ and $B^{2}=-1$ and $A \cdot B=0$. Reading off rotation numbers we see that $c_{1}(A)=-3$ and $c_{1}(B)=-5$ so that $c_{1}$ is Poincaré dual to $-3 A+5 B$ and $c_{1}^{2}=-16$. Also, $\sigma=0, \chi=3$ and the number of left-handed Dehn twists is $q=4$. Thus a final calculation gives $d_{3}=\left(c_{1}^{2}-2 \chi-3 \sigma\right) / 4+q=(-16-6) / 4+4=-3 / 2$.

Next simplest case: $X=S^{1} \times B^{3}$ and $C=S^{1} \times\{0\} \quad$ Now we need to construct a convex BLF on $X=S^{1} \times B^{3}$ inducing a given homotopy class of plane fields on $S^{1} \times S^{2}$. By the comments earlier on the 3 -dimensional invariant and connected sums of broken Lefschetz fibrations, if we get the 2-dimensional invariant correct then we can use the case above for $B^{4}$ to get the 3-dimensional invariant correct (and overtwistedness will come from the overtwistedness on $\left.B^{4}\right)$. Thus we need to construct a convex BLF $f$ on $X$ such that $c_{1}\left(\left.j(f)\right|_{\partial X}\right)=2 n$ for any given $n \in \mathbb{Z}=H^{2}\left(S^{1} \times S^{2}\right)$. Note that we do not need to worry about the potential sign ambiguities associated with the identification of $\mathbb{Z}$ with $H^{2}\left(S^{1} \times S^{2}\right)$ because there is an orientation-preserving automorphism of $S^{1} \times B^{3}$ which induces multiplication by -1 on $H^{2}\left(S^{1} \times S^{2}\right)$. 

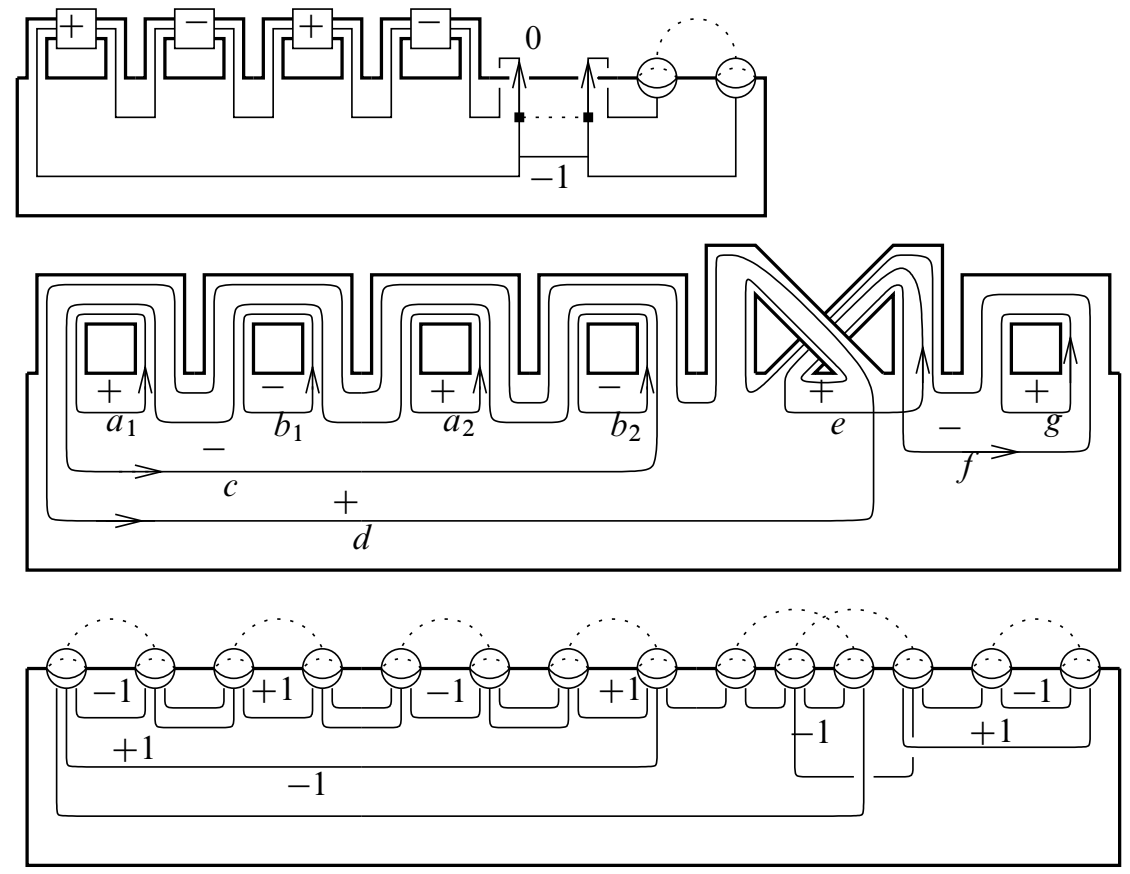

Figure 12: A broken Lefschetz fibration on $B^{4}$ for the case $n=-1$, ie $d_{3}=-3 / 2$.

So we can simplify the problem slightly to say that, given any non-negative integer $n$, we should construct $f$ so that $\left|c_{1}\left(z\left(\left.f\right|_{\partial X}\right)\right)\right|=2 n$. If $n=0$ the fibration is $S^{1} \times[0,1] \times D^{2} \rightarrow D^{2}$.

Figure 13 is an explicit example for $n>0$, and should be interpreted as follows: The left diagram is a (round) handlebody decomposition of $S^{1} \times B^{3}$, starting with $S^{3}=\partial B^{4}$ with the binding of the standard open book indicated by the heavy lines, and involving $2 n$ 1-handles strung on this binding, $n$ round 1 -handles each wrapping once around the binding, $n$ 2-handles with framing pf -1 each running over one of the round 1 -handles, and $n-12$-handles with framing pf -1 each connecting one 1-handle to another. (The framings are not indicated in the diagram.) This describes a convex BLF $f: X \rightarrow D^{2}$. Again, using the techniques of Lemma 5.4, we compute the monodromy which is indicated in the right diagram. Here the curves indicate Dehn twists but their handedness is not indicated in the diagram. Their handedness is as follows: The curves labelled $a_{i}, c_{i}, e_{i}$ and $f_{i}$ are right-handed Dehn twists and the curves labelled $b_{i}$ and $d_{i}$ are left-handed Dehn twists. 

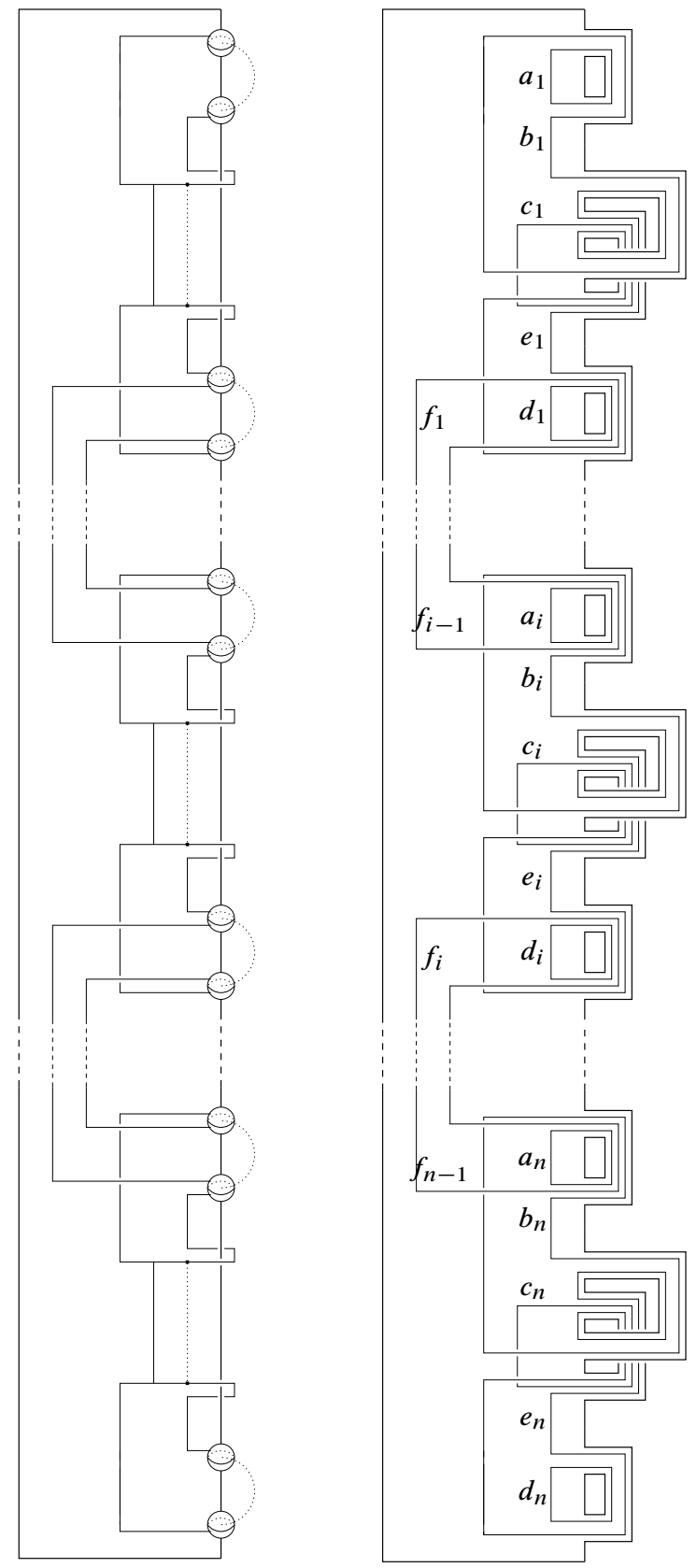

Figure 13: A broken Lefschetz fibration on $S^{1} \times B^{3}$. 
We will now compute $c=c_{1}\left(z\left(\left.f\right|_{\partial X}\right)\right)$. Orient each curve (in the diagram on the right in Figure 13) so that its leftmost straight line segment is oriented downwards. With this orientation, we have that $\operatorname{rot}\left(e_{i}\right)=-1$ and $\operatorname{rot}\left(a_{i}\right)=\operatorname{rot}\left(b_{i}\right)=\operatorname{rot}\left(c_{i}\right)=$ $\operatorname{rot}\left(d_{i}\right)=\operatorname{rot}\left(f_{i}\right)=+1$. Now we convert the monodromy diagram into a handlebody decomposition of a new 4-manifold so that the 1-handles of the page become 4dimensional 1-handles and the Dehn twist curves become attaching circles for 2handles, with $a_{i}, c_{i}, e_{i}$ and $f_{i}$ framed -1 and $b_{i}$ and $d_{i}$ framed +1 . Then we see that $H_{2}$ is generated by $A_{1}, \ldots, A_{n}$ and $F_{1}, \ldots, F_{n-1}$ where $A_{i}=b_{i}-a_{i}+e_{i}-d_{i}$ and $F_{i}=f_{i}-d_{i}-a_{i+1}$. All intersections between generators are 0 except for $F_{i} \cdot F_{i}=-1$, $A_{i} \cdot F_{i}=+1$ and $A_{i+1} \cdot F_{i}=-1$. Thus $H_{2}$ of the boundary $S^{1} \times S^{2}$ is generated by $A=A_{1}+A_{2}+\cdots+A_{n}$. Finally, we evaluate $c$ on $A$ using the rotation numbers above to get $c(A)=c\left(A_{1}\right)+c\left(A_{2}\right)+\cdots+c\left(A_{n}\right)$, with $c\left(A_{i}\right)=\operatorname{rot}\left(b_{i}\right)-\operatorname{rot}\left(a_{i}\right)+$ $\operatorname{rot}\left(e_{i}\right)-\operatorname{rot}\left(d_{i}\right)=-2$, so that $c(A)=-2 n$. Thus $|c|=2 n \in H^{2}\left(S^{1} \times S^{2}\right)=\mathbb{Z}$.

General case Now we are given a general 2-handlebody $X$ and a collection $C \subset$ $(X \backslash \partial X)$ of $m$ points and $n$ circles. Choose a handlebody decomposition of $X$ involving $m 0$-handles, $n$ copies of $S^{1} \times B^{3}$ (ie $n 0$-handles and $n 1$-handles), and then some more 1-and 2-handles, so that each of the $m$ points is contained in one of the $m 0$-handles and each of the $n$ circles is the core of one of the copies of $S^{1} \times B^{3}$. We apply the above cases to each of the $m 0$-handles and each of the $n$ copies of $S^{1} \times B^{3}$. This gives us a convex BLF on $N=\left(\amalg^{m} B^{4}\right) \amalg\left(\amalg^{n} S^{1} \times B^{3}\right)$ with the correct almost complex structure, to which we must add 1 - and 2 -handles. Since there is only one way to extend an almost complex structure across a 1-handle, we can attach the 1-handles as 1-handles strung along the binding of the induced open book, and the BLF then extends across the 1 -handles. At this stage we have constructed a convex BLF $f_{(1)}$ on the 1-handlebody $X_{(1)} \supset N \supset C$, such that $R_{f_{(1)}} \subset N$, such that any almost complex structure $J_{(1)} \in j\left(f_{(1)}\right)$ is homotopic to $J$ on $X_{(1)} \backslash N$, and such that the positive contact structure $\xi_{(1)}$ supported by $\left.f_{(1)}\right|_{\partial X_{(1)}}$ is overtwisted.

The remaining task is to extend this BLF across the 2-handles, getting the almost complex structure correct. One approach is to use the general principle that, once one is free to plumb on right- and left-handed Hopf bands, one can arrange for any framings and rotation numbers of knots lying in pages of open books; this principle is exemplified in Etnyre and Fuller [12]. However, to use this principle, one should note that each right-handed Hopf band introduces a new round 1-handle singularity in a ball across which the almost complex structure does not extend, and so to keep the almost complex structure correct one then needs to compensate by performing a connected sum with an appropriately chosen BLF on $B^{4}$ as in the first part of this proof. Here we present an easier argument that ultimately relies on the uniqueness part of Giroux's correspondence between OBDs and contact structures. 
We will stabilize the OBD $\left.f_{(1)}\right|_{\partial X_{(1)}}$ on $\partial X_{(1)}$ using only left-handed Hopf bands, so that the attaching maps for the 2 -handles all lie in a page, with handle framings equal to $\mathrm{pf}-1$, and with the rotation numbers relative to some fixed trivialization of $\xi_{(1)}$ correct. (Recall from the discussion preceding this proof that the desired almost complex structure will determine rotation numbers for the attaching circles of the 2handles, and that if these rotation numbers are achieved and we extend the BLF across the 2-handles as in Remark 2.2 then the almost complex structure will be correct.) If we can find such a stabilization, then the stabilizations can be realized using Lemma 4.1, extending $f_{(1)}$ across a collar on $\partial X_{(1)}$ without introducing new round 1-handle singularities, and then $f_{(1)}$ can be extended across the 2-handles as in Remark 2.2.

The existence of this stabilization can be seen as follows: Suppose we are given an overtwisted contact 3-manifold $(M, \xi)$ with a supporting OBD $g: M \rightarrow B^{2}$. Because $\xi$ is overtwisted, any framed link $L$ in $(M, \xi)$ can be isotoped to a Legendrian link with the given framing equal to one less than the contact framing (ie framing equal to tb -1 ), and with any specified rotation number relative to a fixed trivialization of $\xi$. (See in [16, Lemma 11], for example.) Now note that, for any Legendrian link $L$ in $(M, \xi)$ there is an OBD $g^{\prime}$ on $M$ supporting $\xi$ such that $L$ lies in a page of $g^{\prime}$ (and therefore the contact framing is the page framing). This can be seen by first noting that it is true in $S^{3}$ with the standard tight contact structure $\xi_{0}$, as proved in [3]. Then note that $(M, \xi)$ is the result of \pm 1 contact surgery (as in [8]) on some Legendrian link $L^{\prime}$ in $\left(S^{3}, \xi_{0}\right)$, and that there is another Legendrian link $L^{\prime \prime}$ in $\left(S^{3}, \xi_{0}\right)$ disjoint from $L^{\prime}$ such that, after surgery, $L^{\prime \prime}$ becomes the given link $L$ in $(M, \xi)$. Find the OBD on $S^{3}$ which contains $L^{\prime} \cup L^{\prime \prime}$ in a page, then perform \pm 1 contact surgery on $L^{\prime}$ and note that this produces an OBD on $(M, \xi)$ with $L$ lying in a page. Note also that $L$ then lies in a page of any stabilization of $g^{\prime}$. By Giroux's uniqueness result, $g^{\prime}$ and $g$ have a common stabilization $g^{\prime \prime}$, which therefore contains $L$ in a page. Because $L$ was a fixed Legendrian knot throughout, and because $L$ had the correct contact framing and rotation number to begin with, $L$ has the correct page framing and rotation number at the end.

\section{Proof of the main result}

We need one more trick to complete the proof of our main result, namely the ability to stabilize OBDs on concave boundaries.

Lemma 6.1 Given a concave fibration $f: X^{4} \rightarrow \Sigma$ and an arc $A$ in a page of the induced OBD $f: \partial X \rightarrow B^{2}$. Let $f^{\prime}: \partial X \rightarrow B^{2}$ be the result of positively (resp. negatively) stabilizing this $O B D$ along $A$. Then $f^{\prime}$ extends to a concave fibration 
$f^{\prime}: X \rightarrow \Sigma$ which agrees with $f$ outside a ball neighborhood of $A$, inside which the only singularities are a round 1 -handle singularity and a Lefschetz (resp. anti-Lefschetz) singularity.

Proof Let $\alpha_{0}$ and $\alpha_{1}$ be the endpoints of $A$. Now add a cancelling pair of $1-$ and 2-handles where the feet of the one handle lie on $A$ near $\alpha_{0}$ and $\alpha_{1}$ and where the attaching map of the 2-handle goes over the 1 -handle once and follows $A$, with framing $\mathrm{pf}-1$ (resp. $\mathrm{pf}+1$ ).

Now add a cancelling 2-3-handle pair and proceed as in the proof of Lemma 4.5 to turn the one handle into a round 1-handle (adding a $T^{2}$ to the fiber) whereupon the 3-handle removes an annulus from the page leaving the following: the page has had a 1 -handle attached with a Dehn twist along $\alpha$, right (left) handed if the framing was page framing $-1(+1)$. This is exactly the stabilization that was desired.

Proof of Theorem 1.1 Split $X$ as $A \cup B$ where $A$ is the result of attaching some number of 1-handles to a neighborhood $F \times B^{2}$ of $F$ and $B$ is a 2-handlebody. Lemma 3.1 gives a concave BLF $f: F \times B^{2} \rightarrow S^{2}$, which we extend across the rest of the 1-handles of $A$ using Lemma 4.5. Use Lemma 6.1 to positively stabilize the induced OBD $\left.f\right|_{\partial A}: \partial A \rightarrow B^{2}$. Now shift attention to $B$, where $\partial B=-\partial A$, and consider the problem of extending the given OBD on $\partial A$ across $B$. First note that the (positive) contact structure supported by this OBD on $\partial B$ is in fact overtwisted, precisely because it resulted from a positive stabilization on $\partial A$, which is a negative stabilization on $\partial B=-\partial A$. Thus Corollary 5.3 gives us a convex BLF $g: B \rightarrow B^{2}$ which induces on OBD on $\partial B$ which is the result of positively stabilizing the given OBD coming from $-\partial A$. Note that at this point we have a concave BLF on $A$ and a convex BLF on $B$, which do not quite match because we need to positively stabilize (in the sense of the orientation coming from $B$ ) the OBD coming from $A$, ie we need to negatively stabilize the OBD on $\partial A$. Thus we use Lemma 6.1 one more time, but finally we are forced to introduce achirality, in order to achieve these negative stabilizations, and then the BALF on $A$ can be glued to the BLF on $B$.

Note that if we could find a trick for negatively stabilizing the concave side without introducing anti-Lefschetz singularities, we would be able to avoid achirality completely. The authors did find some tricks analogous to Lemma 5.4 that work on the concave side, but these always involved extraneous extra positive stabilizations which we could not control.

Proof of addendum to Theorem 1.1 Here we are given the extra data of some 2spheres $S_{1}, \ldots, S_{n}$ which should become sections of the BALF. In this case split $X$ as 
$A \cup B$ where $A$ is $F \times B^{2}$ together with $n$ 2-handles attached along sections $p_{i} \times S^{1}$ of $F \times S^{1}$, and some extra 1-handles so that the complement is a 2-handlebody, and so that the 2-handles give the spheres $S_{i}$. Start with the flat fibration $F \times B^{2} \rightarrow B^{2}$, then attach the section 2-handles as in Remark 2.10, to get concave boundary, and then attach the 1-handles as in Lemma 4.5. This gives the concave piece, and proceed as in the proof above to put a convex BLF on the complement, and to make the open books match by appropriate stabilizations.

To arrange that the round 1-handle singularities all lie over the tropics of Cancer and Capricorn, notice that the only place in our construction where the attaching circles for a round 1-handle might run over another round 1-handle is in the negative stabilizations on the convex side (Lemma 5.4). However, if we do not try to keep the convex side chiral, we can achieve these stabilizations with anti-Lefschetz singularities rather than round 1-handle singularities. Then the round 1-handle singularities on the convex side are independent and therefore can lie over the tropic of Capricorn, and those on the concave side are also independent and can lie over the tropic of Cancer. Finally, on each side, the vanishing cycle 2-handles can always be attached after attaching the round 1-handles, so we can arrange for them to project to the equator.

Remark 6.2 Here we explain our understanding of Perutz's comment mentioned in Remark 1.4 (based on an example in [4]) that, after blowing up, we can in fact get rid of all the anti-Lefschetz singularities. A neighborhood of an anti-Lefschetz singularity is a convex BLF as in Figure 14, where the total 4-manifold is $B^{4}$ represented as a 1-handle and a cancelling 2-handle, and where the OBD on the boundary $S^{3}$ has an annulus as page and a left-handed Dehn twisted about the core of the annulus as monodromy. Figure 15 is a convex BLF where the total 4 -manifold is $\overline{\mathbb{C} P^{2}} \backslash B^{4}$ represented as two 0 -handles and one round 1 -handle. The OBD on the boundary $S^{3}$ is again an annulus with a left-handed Dehn twist about the core. Thus the first BLF can be replaced by the second BLF, changing the 4-manifold by a blowup and replacing the anti-Lefschetz singularity with a round 1-handle singularity.

\section{Questions}

Question 7.1 The most basic question is, "What is the uniqueness theorem?" Many choices are made in the construction of a BALF; if different choices are made, what is the set of moves relating the two BALFs? These should include, for example, the positive and negative stabilizations used to match the convex and concave pieces, and adding cancelling round 1-2-handle pairs. A critical ingredient would be a uniqueness statement for the sequences of stabilizations coming from Giroux's results. 


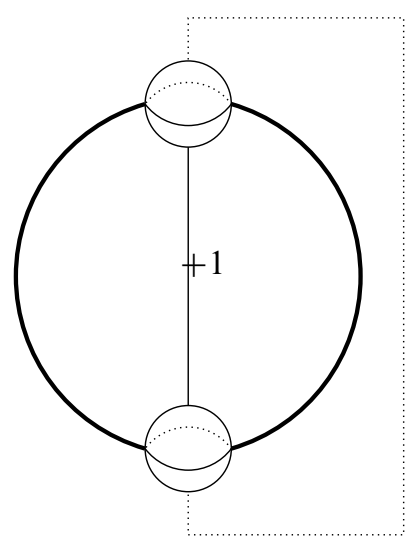

Figure 14: A neighborhood of an anti-Lefschetz singularity. (The bold lines are the binding of the open book.)
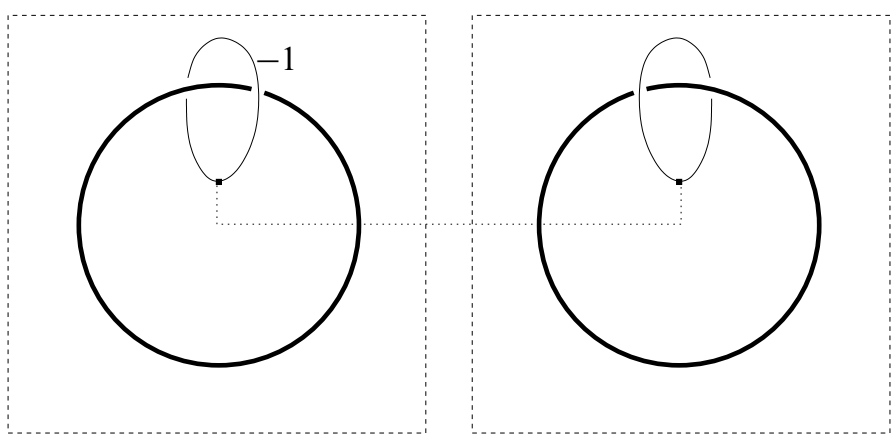

Figure 15: A replacement for the anti-Lefschetz singularity. (Bold lines are binding, and the two dashed squares are the two 0 -handles.)

Question 7.2 Another question is whether achirality can be avoided. By the results in [4], if $b_{2}^{+}(X)>0$ and we blow up enough, then this can be done; but even in this case we do not have a constructive proof.

Achirality could be avoided in general if we could find a way to positively and negatively stabilize the concave side using only Lefschetz and round 1-handle singularities. If this cannot be done, there ought to be an obstruction which lies in the set of equivalence classes of OBDs on connected sums of $S^{1} \times S^{2}$ 's, where the equivalence relation is derived from the basic moves in the uniqueness question above. Even better would be to push this obstruction to the $S^{3}$ boundary of the 4-handle. 
Question 7.3 In [4] it is shown that a BLF supports a near-symplectic form as long as there is a 2-dimensional cohomology class evaluating positively on the fibers. (This is a closed 2-form vanishing identically along the round 1-handle singularities, symplectic in their complement, and satisfying a certain transversality along the circles.) Does this generalize meaningfully to the case of a BALF? What control on the 2-form can we expect near the anti-Lefschetz singularities? Baykur [6] has used ALFs to construct folded symplectic structures.

Question 7.4 To what extent does achirality destroy Perutz's program $[30 ; 31 ; 32]$ to generalize the Donaldson-Smith-Usher [10;36] results relating smooth 4-manifold invariants to counts of multisections of Lefschetz fibrations? Perutz proposes to count multisections of BLFs (some of which may limit on round 1-handle singularities); see also [5].

Question 7.5 A smooth, simply-connected 5-dimensional $h$-cobordism is a product off of a contractible manifold, which is an $h$-cobordism between two contractible 4-manifolds, $A_{0}$ and $A_{1}$ (Curtis et al [7], Kirby [24] and Matveyev [29]). These 4manifolds, called Akbulut's corks, are constructed from a symmetric link of 0-framed unknots by changing half the unknots to 1 -handles (replacing the 0 by a dot), or the other half to 1-handles. There is an involution $h: \partial A_{0} \rightarrow \partial A_{1}=\partial A_{0}$ which does not extend to a diffeomorphism from $A_{0}$ to $A_{1}$.

The question here is whether each of $A_{0}$ and $A_{1}$ are convex $\mathrm{B}(\mathrm{A}) \mathrm{LFs}$ such that the involution $h$ preserves the induced OBD on the boundary, so that the process of surgering out $A_{0}$ and replacing with $A_{1}$ can be carried out on closed B(A)LFs without changing the fibration outside $A_{0}$.

\section{References}

[1] S Akbulut, R Kirby, An exotic involution of $S^{4}$, Topology 18 (1979) 75-81 MR528237

[2] S Akbulut, R Kirby, A potential smooth counterexample in dimension 4 to the Poincaré conjecture, the Schoenflies conjecture, and the Andrews-Curtis conjecture, Topology 24 (1985) 375-390 MR816520

[3] S Akbulut, B Ozbagci, Lefschetz fibrations on compact Stein surfaces, Geom. Topol. 5 (2001) 319-334 MR1825664

[4] D Auroux, S K Donaldson, L Katzarkov, Singular Lefschetz pencils, Geom. Topol. 9 (2005) 1043-1114 MR2140998

[5] R İ Baykur, Near-symplectic broken Lefschetz fibrations and (smooth) invariants of 4-manifolds in preparation 
[6] R İ Baykur, Kähler decomposition of 4-manifolds, Algebr. Geom. Topol. 6 (2006) 1239-1265 MR2253445

[7] C L Curtis, MH Freedman, W C Hsiang, R Stong, A decomposition theorem for h-cobordant smooth simply-connected compact 4-manifolds, Invent. Math. 123 (1996) 343-348 MR1374205

[8] F Ding, H Geiges, A I Stipsicz, Surgery diagrams for contact 3-manifolds, Turkish J. Math. 28 (2004) 41-74 MR2056760

[9] S K Donaldson, Lefschetz pencils on symplectic manifolds, J. Differential Geom. 53 (1999) 205-236 MR1802722

[10] S Donaldson, I Smith, Lefschetz pencils and the canonical class for symplectic fourmanifolds, Topology 42 (2003) 743-785 MR1958528

[11] Y Eliashberg, Classification of overtwisted contact structures on 3-manifolds, Invent. Math. 98 (1989) 623-637 MR1022310

[12] J B Etnyre, T Fuller, Realizing 4-manifolds as achiral Lefschetz fibrations, Int. Math. Res. Not. (2006) Art. ID 70272, 21 MR2219214

[13] J B Etnyre, B Ozbagci, Invariants of contact structures from open books arXiv: math.GT/0605441

[14] M H Freedman, The topology of four-dimensional manifolds, J. Differential Geom. 17 (1982) 357-453 MR679066

[15] D T Gay, Open books and configurations of symplectic surfaces, Algebr. Geom. Topol. 3 (2003) 569-586 MR1997330

[16] D T Gay, R Kirby, Constructing symplectic forms on 4-manifolds which vanish on circles, Geom. Topol. 8 (2004) 743-777 MR2057780

[17] E Giroux, Géométrie de contact: de la dimension trois vers les dimensions supérieures, from: "Proceedings of the International Congress of Mathematicians, Vol. II (Beijing, 2002)”, Higher Ed. Press, Beijing (2002) 405-414 MR1957051

[18] R E Gompf, Killing the Akbulut-Kirby 4-sphere, with relevance to the Andrews-Curtis and Schoenflies problems, Topology 30 (1991) 97-115 MR1081936

[19] R E Gompf, Handlebody construction of Stein surfaces, Ann. of Math. (2) 148 (1998) 619-693 MR1668563

[20] R E Gompf, A I Stipsicz, 4-manifolds and Kirby calculus, Graduate Studies in Mathematics 20, American Mathematical Society, Providence, RI (1999) MR1707327

[21] J Harer, Pencils of curves on 4-manifolds, PhD thesis, University of California, Berkeley (1979)

[22] J Harer, How to construct all fibered knots and links, Topology 21 (1982) 263-280 MR649758 
[23] C Hog-Angeloni, W Metzler, The Andrews-Curtis conjecture and its generalizations, from: "Two-dimensional homotopy and combinatorial group theory", London Math. Soc. Lecture Note Ser. 197, Cambridge Univ. Press, Cambridge (1993) 365-380 MR1279186

[24] R Kirby, Akbulut's corks and h-cobordisms of smooth, simply connected 4-manifolds, Turkish J. Math. 20 (1996) 85-93 MR1392665

[25] R Kirby, Problems in low-dimensional topology, from: "Geometric topology (Athens, GA, 1993)”, AMS/IP Stud. Adv. Math. 2, Amer. Math. Soc., Providence, RI (1997) 35-473 MR1470751

[26] F Laudenbach, V Poénaru, A note on 4-dimensional handlebodies, Bull. Soc. Math. France 100 (1972) 337-344 MR0317343

[27] W B R Lickorish, A representation of orientable combinatorial 3-manifolds, Ann. of Math. (2) 76 (1962) 531-540 MR0151948

[28] A Loi, R Piergallini, Compact Stein surfaces with boundary as branched covers of $B^{4}$, Invent. Math. 143 (2001) 325-348 MR1835390

[29] R Matveyev, A decomposition of smooth simply-connected h-cobordant 4-manifolds, J. Differential Geom. 44 (1996) 571-582 MR1431006

[30] T Perutz, Surface-fibrations, four-manifolds, and symplectic Floer homology, $\mathrm{PhD}$ thesis, University of London (2005)

[31] T Perutz, Lagrangian matching invariants for fibred four-manifolds. I, Geom. Topol. 11 (2007) 759-828 MR2302502 arXiv:math.SG/0606061

[32] T Perutz, Lagrangian matching invariants for fibred four-manifolds. II arXiv: math.SG/0606062

[33] C H Taubes, Pseudoholomorphic punctured spheres in $\mathbb{R} \times\left(S^{1} \times S^{2}\right)$ : moduli space parametrizations, Geom. Topol. 10 (2006) 1855-2054 MR2284051

[34] C H Taubes, Pseudoholomorphic punctured spheres in $\mathbb{R} \times\left(S^{1} \times S^{2}\right)$ : properties and existence, Geom. Topol. 10 (2006) 785-928 MR2240906

[35] I Torisu, Convex contact structures and fibered links in 3-manifolds, Internat. Math. Res. Notices (2000) 441-454 MR1756943

[36] M Usher, The Gromov invariant and the Donaldson-Smith standard surface count, Geom. Topol. 8 (2004) 565-610 MR2057774

Department of Mathematics and Applied Mathematics, University of Cape Town,

Private Bag X3, Rondebosch 7701, South Africa

University of California, Berkeley, CA 94720, USA

David.Gay@uct.ac.za, kirby@math.berkeley.edu

Proposed: Simon Donaldson

Seconded: Ronald J Stern, Ron Fintushel
Received: 4th January 2007

Revised: 2nd July 2007 